\title{
Exploring current practices in pediatric ARV rollout and integration with early childhood programs in South Africa: A rapid situation analysis
}

Desiree Michaels

Brian Eley

Lewis Ndhlovu

Population Council

Naomi Rutenberg

Population Council

Follow this and additional works at: https://knowledgecommons.popcouncil.org/departments_sbsr-hiv

Part of the Demography, Population, and Ecology Commons, Health Policy Commons, Immune System Diseases Commons, International Public Health Commons, Maternal and Child Health Commons, Medicine and Health Commons, Public Health Education and Promotion Commons, and the Virus Diseases Commons How does access to this work benefit you? Let us know!

\section{Recommended Citation}

Michaels, Desiree, Brian Eley, Lewis Ndhlovu, and Naomi Rutenberg. 2006. "Exploring current practices in pediatric ARV rollout and integration with early childhood programs in South Africa: A rapid situation analysis," Horizons Final Report. Washington, DC: Population Council. 
Exploring Current Practices in Pediatric ARV Rollout and Integration with Early Childhood Programs in South Africa: A Rapid Situation Analysis
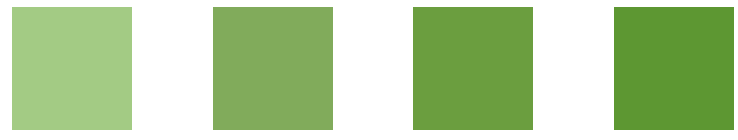

University of Cape Town Horizons Program 


\section{Exploring Current Practices in Pediatric ARV Rollout and I ntegration with Early Childhood Programs in South Africa: A Rapid Situational Analysis}

Compiled by Desireé Michaels, Brian Eley,

Lewis Ndhlovu, and Naomi Rutenberg 


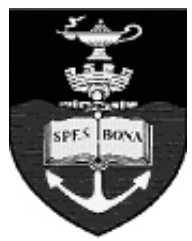

The mission of the University of Cape Town School of Public Health is to achieve excellence in research, education and service in public health and family medicine, in pursuit of equity and social justice. The Infectious Diseases Epidemiology Unit is involved in a wide range of interdisciplinary research and teaching activities spanning surveillance, HIV-prevention clinical trials, clinical epidemiology of tuberculosis and HIV therapy, socio-behavioural and health systems research. http://www.epi.uct.ac.za/

The mission of the School of Child and Adolescent Health, University of Cape Town is the promotion, restoration and maintenance of the health and well-being of children and adolescents. This is achieved through excellence in service, teaching, leadership and research at all levels of health care. The Paediatric Infectious Diseases Unit at Red Cross Children's Hospital plays a leading role in advancing the care of HIV-infected children.

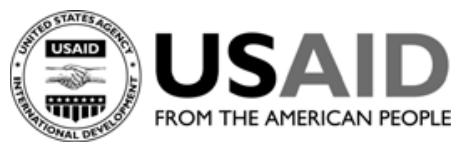

This project was funded by the Horizons Program. Horizons is implemented by the Population Council in collaboration with the International Center for Research on Women, International HIV/AIDS Alliance, PATH, Tulane University, Family Health International, and Johns Hopkins University. Horizons is funded by the President's Emergency Plan for AIDS Relief through the U.S. Agency for International Development, under the terms of HRN-A-000-97-00012-00. The opinions expressed herein are those of the authors and do not necessarily reflect the views of the U.S. Agency for International Development.

Published in June 2006.

\section{(2) Population Council}

The Population Council is an international, non-profit, nongovernmental institution that seeks to improve the well-being and reproductive health of current and future generations around the world and to help achieve a humane, equitable, and sustainable balance between people and resources. The Council conducts biomedical, social science, and public health research and helps build research capacities in developing countries. Established in 1952, the Council is governed by an international board of trustees. Its New York headquarters supports a global network of country offices.

Copyright 2006 The Population Council Inc.

Suggested citation: Michaels, Desireé, Brian Eley, Lewis Ndhlovu, and Naomi Rutenberg. 2006. "Exploring current practices in pediatric ARV rollout and integration with early childhood programs in South Africa: A rapid situational analysis," Horizons Final Report. Washington, DC: Population Council.

This document may be reproduced in whole or in part without permission of the Population Council provided full source citation is given and the reproduction is not for commercial purposes. 


\section{Acknowledgements}

\section{I nvestigators}

\section{University of Cape Town}

Desireé Michaels (Public Health \& Family Med)

Brian Eley (School of Child and Adolescent Health)

\section{Population Council}

Lewis Ndhlovu

Naomi Rutenberg

\section{Site Collaborators}

$\begin{array}{lll}\begin{array}{l}\text { Eastern Cape } \\ \text { MSF }- \text { Lusikisiki }\end{array} & - & \text { Dr Herman Reuter } \\ \quad \text { East London (CM) } & - & \text { Dr Gerald Boon } \\ \begin{array}{l}\text { Gauteng } \\ \quad \text { Baragwaneth Hospital }\end{array} & - & \text { Dr Tammy Myers } \\ \begin{array}{l}\text { Free State } \\ \quad \text { University of Free State }\end{array} & - & \text { Dr Elizabeth Tabane } \\ \begin{array}{l}\text { Kwa-Zulu Natal } \\ \quad \text { McCord Hospital }\end{array} \\ \begin{array}{l}\text { Western Cape } \\ \quad \text { Red Cross Children's Hospital }\end{array} \\ \quad- & \text { Dr Willem Vlok } \\ \quad \text { MSF - Khayelitsha } & - & \text { Dr Brian Eley } \\ \end{array}$

\section{Research Team}

Protocol Development Team - Investigators

Lewis Ndhlovu

Naomi Rutenberg

Desireé Michaels

Brian Eley

Project Manager

Desireé Michaels

\section{Assistant Project Manager \\ Pat Francis}

\section{Administrative Support}

Candice Hickley 
Healthcare Worker Survey Researchers

Washiefa Isaacs

Carol Cragg

Larissa Reader

Caregiver Survey Researchers

Gertrude Qiki

Nontobeko Mvimbi

Glen Mabuza

Nomandla Yako

Data Management \& Analysis Qualitative Data

Desireé Michaels

Pat Francis

Washiefa Isaacs

Nkosinathi Sohaba

Transcriptions

Pat Francis

Washiefa Isaacs

\section{Quantitative Data}

Louis Apicella (Washington)

Lewis Ndhlovu

Washiefa Isaacs

Desireé Michaels

Special thanks to Dr. David Coetzee and Dr. Andrew Boulle (Infectious Diseases Epidemiology Unit) for managerial and technical assistance and to all those who assisted fieldworkers during site visits, especially Merleesa Naidoo (Gauteng); Noxolo and Nomthu (Lusikisiki); Sr. Rolom (Cecilia Makewane); Dianne Potterton (Free State); Lito Gogo (Khayelitsha); and to all health care workers and caregivers who granted interviews.

\section{Stakeholders}

National Department of Health

Provincial Health Departments

Horizons Program, Population Council

Elizabeth Glaser Pediatric AIDS Foundation, SA

Centre for Disease Control, SA

Department of Public Health \& Family Medicine, UCT

School of Child \& Adolescent Health, UCT

\section{Funding Support}

USAID through Horizons/Population Council

Infectious Disease Epidemiology Unit, UCT 


\section{Table of Contents}

\section{Abbreviations and Acronyms}

Executive Summary 1

Introduction

Children in the global ART scale-up $\quad 5$

Study background $\quad 6$

$\begin{array}{ll}\text { Study aims and objectives } & 7\end{array}$

$\begin{array}{ll}\text { Policy Review } & 9\end{array}$

Introduction 9

Entry points into the care delivery system 9

$\begin{array}{ll}\text { Antiretroviral therapy in pediatrics } & 10\end{array}$

$\begin{array}{ll}\text { Nutrition-related interventions } & 10\end{array}$

$\begin{array}{ll}\text { Continuum of care services } & 11\end{array}$

$\begin{array}{ll}\text { Accreditation of service points } & 11\end{array}$

Projected treatment and monitoring costs $\quad 11$

Planning for and monitoring the pediatric ARV rollout $\quad 12$

Conclusion 13

Overview of Methods and Study Sites 14

$\begin{array}{ll}\text { Methods } & 14\end{array}$

Description of sites $\quad 16$

Health provider surveys and interviews 19

Health Care Worker Training, Experience, and 22 Roles in Pediatric ARV Service

Doctors 22

Nurses $\quad 23$

$\begin{array}{ll}\text { Counselors } & 25\end{array}$ 
$\begin{array}{ll}\text { Social workers } & 26\end{array}$

$\begin{array}{ll}\text { Pharmacists } & 26\end{array}$

\section{Current and Optimal Sources of Referral for Children to ARV Treatment $\quad 29$}

Current sources of referral to ARV clinics 29

Optimal sources of referral 29

Major Challenges to Pediatric ARV Rollout 32

Human resource challenges $\quad 32$

Physical and structural challenges $\quad 36$

Policy and procedural challenges $\quad 37$

Socioeconomic challenges $\quad 38$

$\begin{array}{ll}\text { Funding } & 42\end{array}$

Examples of overcoming challenges to the ARV rollout 43

$\begin{array}{lr}\text { Caregiver Survey } & 47\end{array}$

$\begin{array}{ll}\text { Background information } & 47\end{array}$

Access to treatment $\quad 48$

$\begin{array}{ll}\text { Perception of quality of services } & 50\end{array}$

Conclusion and Recommendations 52

Build an effective, comprehensive PMTCT program 53

Identify HIV-infected children

Train health professionals $\quad 53$

Monitor services for HIV-infected children $\quad 54$

Utilize models of care $\quad 54$

Promote collaboration between clinical services and non-clinical governmental 54 sectors, community-based services, and health systems

Meet the special needs of HIV-infected infants

Encourage adherence $\quad 55$

Adolescence: Support the transition from childhood to adulthood 56 
References

Appendix: Site visits-Fieldworker Observations 


\section{Abbreviations and Acronyms}

AIDS - acquired immune deficiency syndrome

ART - antiretroviral therapy

ARV - antiretroviral

ATICC - AIDS Training Information and Counseling Centres

HIV - human immunodeficiency virus

IMCI - integrated management of childhood illness

MSF - Medecins San Frontieres

PMTCT - prevention of mother-to-child transmission

STI - sexually transmitted infection

TB - tuberculosis

UNICEF - United Nations Children's Emergency Fund

USAID - United States Agency for International Development

VCT - voluntary counseling and testing

WHO - World Health Organization 


\section{Executive Summary}

\section{Introduction}

In 2005, more than half a million children died of AIDS, the vast majority of whom lived in the developing world (UNAIDS 2005). In sub-Saharan Africa, AIDS has become one of the leading causes of mortality among children under the age of five years. Yet despite increased availability of antiretroviral therapy (ART), children remain a neglected population group.

In many countries in Africa, there is a lack of experience in the identification, treatment, and management of young children who are HIV-positive, and limited knowledge of how community and health service providers can work together to improve children's access to treatment. As the burden of care for children affected by and infected with HIV is now evident in many countries in Africa, governments must increase their level of response swiftly to deal with the crisis. The goal of providing ART to children is to increase survival and decrease HIV- and AIDS-related morbidity and mortality.

In South Africa, 40 percent of deaths of children less than 5 years of age are attributable to HIV (Dorrington et al. 2004). The South African Cabinet approved a plan for a national HIV treatment program in 2003, the goal of which was to have at least one service delivery point in each district providing treatment by the end of March 2004. Initial treatment sites were identified and inspected under the auspices of the Department of Health $(\mathrm{DOH})$ in terms of meeting a set of minimum criteria for service delivery. Once these service delivery points are deemed to be functioning adequately, the rollout process will cascade to lower levels as the government's experience improves.

This report presents the results of a rapid situational analysis of the pediatric rollout in South Africa. The study provides much needed information on critical issues of pediatric HIV care, especially regarding health service and contextual issues surrounding the expansion of access to treatment for HIV-infected children, and key factors that facilitate sustainability of treatment by young children.

\section{Aims}

"Exploring current practices in pediatric ARV rollout and integration with early childhood programs in South Africa: A rapid situational analysis" is a collaborative research project between the Horizons Program of the Population Council and the University of Cape Town. The Infectious Diseases Epidemiology Unit of the University of Cape Town provided infrastructure support and cost sharing for the project.

The aims of the study were to identify successful program strategies in pediatric HIV treatment in South Africa and to determine priority knowledge gaps to be addressed by operations research.

\section{Specific objectives}

- Identify critical components of pediatric HIV treatment services.

- Determine ways in which children are currently enrolled into ART programs. 
- Identify referral links to ART programs for children.

- Identify factors that influence access to treatment and adherence for children.

- Examine the barriers to treatment and issues of equity that affect children.

- Describe how challenges regarding ART service delivery to children have been overcome.

- Look at the convergence of practice and national policy and legislation with respect to health care for HIV-infected children.

- Make policy and program recommendations to support and enhance national and provincial rollout of pediatric HIV care.

\section{Methodology}

The ARV programs at 16 institutions in five provinces of South Africa were visited between 11 April 2005 and 21 June 2005. Qualitative and quantitative approaches were used to collect data to describe ARV programs at these institutions. Activities at all facilities were observed during clinic days, various categories of health workers were interviewed using semi-structured questionnaires, structured questionnaires were administered to caregivers, and open-ended interviews were conducted with facility managers. In all but two instances, all interviews with health care workers were tape-recorded. Extensive field notes were compiled during clinic visits and interviews. A total of 7 facility managers at pediatric ARV clinics, 67 other categories of health care workers, and 126 caregivers were interviewed.

\section{Study Sites}

The 16 pediatric ARV sites were grouped into seven clusters of sites. They varied in their overall character and location within the health system, e.g., a pediatric clinic at a tertiary institution in an urban setting and a combined adult and pediatric clinic at a primary level rural facility.

As of 31 March 2005 the 16 sites were treating approximately 1,300 children on ART. About 60 percent of these children were under six years of age. Only 2 of the 16 sites reported having waiting lists of children requiring ART. Site visits and field observation revealed that a variety of locally adapted treatment models are required to meet the needs of infected children and their families in South Africa given the varying strengths and limitations in the health system in different parts of the country.

\section{Policy Review}

The policy review focused on "The operational plan for comprehensive HIV and AIDS care, management and treatment for South Africa, November 2003." Other key national documents referenced included "Strategic priorities for the national health system 2004-2009," (South Africa Department of Health 2003) "Policy guidelines for youth and adolescent health," (South Africa Department of Health 2001), and "Policy framework for non-communicable chronic conditions in children," (South Africa Department of Health 2002).

The review showed that current policy documents cover a wide range of services and interventions for HIV-infected children. However, existing policies are scattered over several documents, are not uniform 
in their presentation, are underdeveloped in certain areas (e.g., lack of specific recommendations for managing infected infants or treating infected adolescents and youth and the care of children without reliable caregivers), and therefore do not clearly facilitate the development of comprehensive services for HIV-exposed and infected children.

\section{Key Results}

\section{Health worker training, roles, and experience}

No standardized or coordinated training program on the management of pediatric HIV and AIDS is currently available to medical personnel in South Africa. Most doctors reported having had no formal training in the area; instead, they have had a range of self-training and mentorship training. Nurses also had varying levels of HIV management training, the majority of which was received from doctors at facilities. Their roles varied across study sites, with some more involved in patient administration and ARV-related activities than others. Generally, pharmacists across the board played an enormous role in adherence monitoring and counseling in addition to maintaining stock levels of ARVs.

\section{Referral sources}

The survey findings indicate that the majority of children were referred for treatment by primary level facilities and in-patient wards at hospitals. Very few patients are referred directly from prevention of mother-to-child transmission (PMTCT) or voluntary counseling and testing (VCT) services. As a result, many of the children are clinically unwell when they begin treatment. Improved linkages with PMTCT programs and improved blood-taking skills at primary level facilities are essential for early identification of children who need treatment.

\section{Health care worker perceptions on pediatric ARV rollout}

Major challenges to the pediatric rollout as perceived by doctors, nurses, counselors, social workers, and pharmacists included clinic space constraints, lack of adequately trained staff, lack of clinical capacity and a "fear of treating children." In particular, the need for nurses to be skilled in taking blood from children was cited. Doctors and nurses also noted gaps in the health system, particularly between the PMTCT program and well-baby clinics.

Other concerns included fewer drug options for children, lack of services for adolescents, widespread poverty and unemployment and the impact of these factors on health care, transport and distance barriers to accessing pediatric HIV care, and the lack of community awareness about ARV services for children and the benefits of such services for infected children. Counselors and pharmacists expressed other concerns surrounding community awareness and support for children with HIV and mentioned issues with medications including dosing, maintaining stock levels and monitoring adherence. Many health care workers cited the emotional challenge of working in the currently constrained system. 


\section{Caregivers survey}

Of the 126 caregivers interviewed, approximately half were from either Baragwaneth Hospital or Red Cross Children's Hospital, the sites with the largest pediatric ARV programs. The primary caregivers were mainly biological mothers, grandmothers, and aunts. Most of the children had been referred from a community clinic to the ARV site, and had been tested for HIV infection because they were either chronically ill or hospitalised. Only 5 percent had been tested through the PMTCT program. Adherence to the medications was generally good: 96 percent of children had received their medication on the morning of the interview; however, 15 percent had missed one or more doses in the previous week. Generally, the caregivers were very satisfied with the quality of the service they received.

\section{Fieldworker observations}

In addition to conducting interviews, researchers also recorded their observations of the study sites in detailed field notes taken during visits (see Appendix). The notes include descriptions of the clinics and pharmacies, staff, and overall functioning of the sites. Although these narratives are impressionistic, they offer additional insight into the environment health workers face during the ARV rollout, and provide details that supplement the information offered during interviews. Based on fieldworker observation, the human factor was clearly the most critical component of a successful pediatric site. Dedicated doctors who forge links and partnerships with other health care workers and organizations to successfully start a service, sometimes with little or no backing, demonstrate how individuals are capable of making a difference.

\section{Conclusion and Recommendations}

This study shows that ARV programs that are treating children successfully vary according to local circumstances. Several components are critical for the success of pediatric ARV programs including child-oriented human resources, technical proficiency particularly relating to obtaining blood samples from young children, and adequate pharmacy stocks of ARVs. Despite having successful pediatric programs, the institutions surveyed identified a large number of concerns and challenges that need to be overcome in order to improve care for infected children. This will require action from key individuals at institutions, and in provincial and national HIV directorates.

Specific recommendations to policymakers include ensuring children are placed on the ARV rollout agenda by including child specific monitoring indicators creating a comprehensive PMTCT program and encouraging early identification of HIV-infected children. A successful pediatric ARV rollout requires additional, standardized training of health professionals, upgrading of nurses' skills at the primary level to identify and care for children with HIV, increased collaboration between clinical services, and raised community awareness. There is also a need to address the special needs of infants with HIV and adolescents as they transition from childhood to adulthood. 


\section{I ntroduction}

\section{Children in the Global ART Scale-up}

According to UNAIDS estimates, approximately 570,000 children died of AIDS in 2005 the vast majority of whom lived in the developing world(UNAIDS 2005). In sub-Saharan Africa, AIDS has become one of the leading causes of mortality among children under the age of five years. Sadly, in resource-poor settings, prevention of mother-to-child transmission (PMTCT) of HIV remains a challenge, and antiretroviral treatment (ART) is not reaching children. In contrast, in resource-rich countries the rate of vertical transmission has declined, access to ART has increased, and children have responded well to treatment with prolonged, healthier lives including improved growth and cognitive development. Despite this evidence, research into the delivery of pediatric HIV treatment of children under the age of 15 in resource-limited settings has lagged behind research in the adult population.

Increased affordability of ARVs and demonstrated feasibility of administering these drugs has led to a rapid expansion in scaling up access to ART in a number of developing countries. Country effort has been supported by the WHO's ambitious plan to provide treatment to 3 million people with HIV in developing countries by the year 2005 (WHO 2003). The U. S. government made a similar commitment; on February 2004, the State Department Office of the Global AIDS Coordinator released a five-year global HIV strategy known as the President's Emergency Plan for AIDS Relief (PEPFAR). The strategy provides a means of spending approximately USD 15 billion to prevent 7 million new HIV infections, to treat 2 million people with HIV, and to care for 10 million HIV-infected persons and those orphaned by AIDS (Office of the Global AIDS Coordinator 2004).

Adults have largely benefited from the treatment initiatives while children under the age of five have been ignored or excluded. According to UNICEF, less than 10 percent of pregnant women access PMTCT interventions, less than 5 percent of children who need ARVS are receiving them, and approximately 1 percent of children born to HIV-infected women are receiving cotrimoxazole prophylaxis (Luo 2005).

The burden of care for children infected with HIV is now evident in many countries in Africa and governments have to increase their level of response swiftly in order to avert a disaster situation. This involves providing ART to children to increase survival and decrease HIV-related morbidity and mortality. According to Watson, et al (1999) our "current understanding of the natural history of HIV infection and the response to treatment [in children] indicates that only high level suppression of viral replication by a HAART regimen offers the potential for long term survival" (p. 687).

HIV prevention efforts in infants (less than one year old) and children ( 1 to 5 years) have been centered on PMTCT programs in developing countries. These programs focus on the prevention of vertical transmission of HIV during delivery and through breast milk, not treatment. More recently, there have been calls to scale up and improve PMTCT programs, adding HIV care for the infants that become infected despite the intervention as well as for their parents. HIV care that can improve the quality of life for children infected with HIV includes cotrimoxazole prophylaxis, growth monitoring, nutritional interventions, and treatment of opportunistic infections. The response has been to initiate PMTCT Plus programs that provide HIV care to the family unit. 
However, children receiving ART through PMTCT Plus programs are a small segment of the population in need of treatment. There is a pool of HIV-positive children living in communities who do not have access to ART for different reasons, including limited availability of PMTCT and PMTCT Plus interventions, limited availability of ART, caregivers' ignorance of HIV status of children, and lack of programs assisting in access to ART. These children would normally first come to the attention of health care providers when they are ill and present themselves at health facilities with symptoms of HIV infection.

\section{South African estimates of treatment need and ARV rollout}

In South Africa 40 percent of deaths in children under 5 years of age are attributable to HIV (Dorrington et al. 2004). Studies by van Kooten Niekerk et al (2005) and Eley et al (2004) demonstrate that ART is effective in decreasing morbidity and mortality in the pediatric population in the South African context.

At present the total number of children on ART in South Africa is not known, but the estimate is currently 15,000. Similarly, the total number of children requiring antiretroviral therapy at present is not known.

Based on the size of the pediatric epidemic in South Africa as estimated by the Medical Research Council (MRC), some academics have suggested that 20 percent of the total number of infected children or approximately 50,000 children, currently need ART (Centre for Actuarial Research and South African Medical Research Council 2004). Because HIV infection progresses far more rapidly in children than in adults, and with more than 50 percent of perinatally infected children dying before their second birthday in Africa, this figure is probably an underestimation of the true proportion of children that should be on ART in South Africa.

The South African Cabinet approved a plan for a national HIV treatment program in 2003 (South Africa Department of Health 2003). The goal of the plan was for at least one service delivery point in each district to be able to provide treatment at first, followed by continued rollout of services. In preparation for ARV rollout, the government drafted guidelines to assist service providers in the management of patients on ART. The guidelines, based on those formulated by WHO, provide a detailed description of administration of ART in adults and children, management of adverse events in these two groups, and adherence to therapy, including the management of post-exposure prophylaxis. The goals of the ARV program, how patients are to be selected for ARVs, and what regimens are to be administered are detailed in the document. Tuberculosis (TB), which commonly coexists in patients infected with HIV, is also discussed. The national ARV treatment guidelines serve as the minimum standards to be followed by service providers. However, some provinces, such as Western Cape, have gone ahead to develop their own simplified guidelines based on those provided by the National Department of Health.

\section{Study Background}

This study included three activities: a consultative workshop, policy review, and rapid situational analysis. The consultative workshop with expert practitioners and stakeholders was held 21 January 2005 in Johannesburg. The purpose of the meeting was to identify key issues in access to pediatric treatment, to introduce the study to site managers, to familiarize the study investigators with the activities and context of each site, and to obtain input and recommendations regarding the study design and instruments (Horizons 2005). 
The policy review focused on the South African government's "Operational plan for comprehensive HIV and AIDS Care, Management and Treatment for South Africa," (South Africa Department of Health 2003) and other key policy documents. The review includes a summary and discussion of some of the limitations of the pediatric HIV care guidelines found in these documents and how they impact on the treatment of HIV-infected children.

The rapid situational analysis of the pediatric rollout of ART in South Africa documents what is happening on the ground: the challenges and lessons learned at key pediatric HIV care sites. Based on reports from a number of managers of sites that were the first to initiate pediatric HIV care, the initial pediatric ARV rollout has resulted in a significant number of children on treatment. Based on reports from participants at the workshop, at study sites in Free State, Gauteng, and Eastern Cape public health services; Red Cross Hospital in Cape Town (Western Cape); the MSF program in Khayelitsha and Lusikisiki; and the McCord Hospital in Durban (KwaZulu Natal), more than 2,000 children are receiving ARV treatment. Additional children are receiving HIV care other than ARV treatment. The majority of these children are younger than six years of age, according to the meeting report.

This study takes advantage of the rich experience of the early providers and developers of pediatric HIV care in South Africa to understand the opportunities for and obstacles to accessible, effective pediatric care. South Africa is the ideal setting for the study because of the country's vast experience and resources in the area of pediatric medicine, as well as the fact that it has been at the forefront of the fight against HIV in the region. The study provides much needed information on critical issues of pediatric HIV care, especially regarding health service and contextual issues surrounding the expansion of access of treatment of HIV-infected children and key factors that facilitate adherence to treatment by young children and their families.

With much to learn from the South African experience, it is hoped that the identification of operational barriers will assist in the planning and delivery of rollout programs to reach more children and to ensure that quality and effective services are delivered to them. The study results have direct policy relevance for the government of South Africa and other countries in the region that are seeking to expand the ARV program for children, as well as for assisting PEPFAR to meet its goal of reaching two million people living with HIV with antiretroviral treatment.

\section{Study Aims and Objectives}

The aims of the study were to identify successful program strategies in pediatric HIV treatment in South Africa and to determine priority knowledge gaps to be addressed by operations research. The specific study objectives were to:

- Identify critical components of pediatric HIV treatment services.

- Determine ways in which children are currently enrolled into ART programs.

- Identify referral links to ART programs for children.

- Identify factors influencing access to treatment and adherence for children.

- Examine the barriers to treatment and issues of equity that affect children.

- Describe how challenges regarding ART service delivery to children have been and can be overcome.

- Look at the convergence of practice and national policy and legislation with respect to health care for HIV-infected children. 
- Make policy and program recommendations to support and enhance national and provincial rollout of pediatric HIV care.

This report first presents the findings of the policy review. It then shares the results of the rapid situational analysis, including the challenges to successful pediatric ARV rollout shared by health providers, fieldworker observations, and the findings from the caregiver survey. The report concludes with recommendations for strengthening the provision of treatment to HIV-infected children. 


\section{Policy Review}

\section{Introduction}

Pediatric HIV care is mentioned in two key South African policy documents. The "Strategic priorities for the national health system for the 2004-2009 term" (South Africa Department of Health 2004) sets out the Department of Health's strategic priorities over a five-year period and incorporates a wide range of measures for improving service delivery. Reference is made to HIV infection, TB, and how the two are related. Under the child health section, the recent expansion of PMTCT services is documented. At the time of the review, PMTCT services were available in more than 204 public hospitals and 1,055 community health centers and clinics. Proposed key activities are linked to 10 health priorities listed in the document. Improvement of the management of communicable diseases and non-communicable illnesses is one such priority area. Key activities linked to this priority include improving the management of all HIV-positive children under the age of five years, and accelerating the implementation of the operational plan for the treatment, management, and care of HIV and AIDS.

This latter document, the "Operational plan for comprehensive HIV and AIDS care, management and treatment for South Africa," (South Africa Department of Health 2003) is the main document that provides guidance for the rollout of HIV treatment for adults and children. The Department of Health launched this document in November 2003 following its decision to implement and fund a national ART intervention program in all provinces.

This chapter includes a summary of the pediatric recommendations in the operational plan and some of its limitations, as well as brief reviews of other key policy documents that impact on the treatment of HIVinfected children. This discussion focuses on children under the age of 14 years because treatment protocols for children over 14 years are the same as those for adults. It is recognized, however, that adolescents are not "mini-adults;" rather they have special needs that pose unique challenges for health service delivery in the context of the ARV rollout program.

\section{Entry Points into the Care Delivery System}

Chapter 1 of the operational plan outlines several "entry points" for ARV including voluntary counseling and testing services, PMTCT programs, clinics offering reproductive health and STI services, primary health care clinics, TB clinics, inpatient hospital settings, and prisons. The plan recognizes the complex relationships between various levels of the health care system in the care and treatment of the HIVinfected individual. This is particularly challenging with respect to infants and children. The opportunity for reaching the majority of infants is during the period between birth and 14 weeks (i.e., during the postpartum follow-up period and primary vaccination period). Thereafter, contact between the health services and the child is mostly restricted to episodes of illness and mass immunization campaigns. The plan notes that "strategies for infant diagnostics are currently coordinated by the PMTCT program" and recommends that "a pediatric monitoring task force be established, and charged with coordinating protocols for infant diagnostics and monitoring with the PMTCT program and the NHLS" (South Africa Department of Health 2003, p.170); but is otherwise silent on strategies for strengthening and expanding the testing of HIV-exposed and identification of HIV-infected children. 
In addition, the plan does not refer specifically to any special considerations regarding the counseling needs for testing children of varying ages. As noted by Shungking \& Zampoli (2005), counselors should be skilled to provide age-appropriate counseling to "children that are old enough to understand issues relating to their HIV-status, as well as being able to judge how much information and participation the child is able to handle" (p.25). According to Giese et al. (2003), despite the widespread availability of voluntary counseling and testing (VCT) at health facilities in South Africa, their research across six sites in five provinces indicated that health workers and counselors were not comfortable counseling and supporting children. Instead, their activities were mostly directed toward the accompanying caregiver and they reported that they were not trained nor equipped to counsel children, including teenagers.

\section{Antiretroviral Therapy in Pediatrics}

Pages 35-37 of the operational plan are dedicated to issues regarding ART in children. The areas covered include the following: (1) confirmation of HIV-positive diagnosis (2) guidelines regarding initiation of treatment (3) national pediatric drug regimen protocol (4) use of cotrimoxazole, and (5) Nevirapine resistance monitoring.

However, in the ensuing discussion on "changing or stopping antiretroviral treatment," adverse event reporting, patient-drug readiness training, adherence, and adherence strategies, no reference is made to children or to the specific circumstances regarding children on treatment (e.g., caregiver issues). The issue of pediatric treatment is also notably absent in the section entitled "special considerations" (South Africa Department of Health 2003, p. 40), which includes guidelines for the South African Military Health Service and Correctional Services.

A recent discussion paper on the pediatric ARV rollout in South Africa examining whether the needs of infected children are adequately addressed in the current national plan for comprehensive care and treatment for HIV and AIDS (Shungking and Zampoli 2005) notes that children's issues were not addressed comprehensively but instead restricted to clinical and technical issues. For example,

- Family care or comprehensive considerations of the mother-infant pair is not adequately promoted.

- The plan does not make special provision for children who do not have an identifiable caregiver.

- The plan does not consider the special needs and vulnerabilities of infants, school-going children, and adolescents. However, a separate document that predates the operational plan, "Policy guidelines for youth and adolescent Health (South Africa Department of Health 2001)," addresses some of the needs of HIV-infected adolescents. This document contains comprehensive policy guidelines for South African youth and adolescents aged 10 to 24 years, and acknowledges that adolescents and youth living with HIV/AIDS constitute a vulnerable group. It argues that the lives and well-being of infected youth may be improved if (1) their living environment is non-discriminatory, (2) they have adequate shelter and nutrition, (3) they have access to treatment for opportunistic infections, and (4) they are supported.

\section{Nutrition-related I nterventions}

The plan is clear in its policy regarding nutrition-related interventions for HIV-infected children under 14 years, evidenced by the following statement: "HIV-positive infants and children face a confluence of three powerful nutritional challenges, namely high nutritional needs to sustain their high growth rate, 
rapid progression to AIDS associated with significant wasting; and an immature, compromised immune system, with increased risk for opportunistic infections and diminished nutritional intake. Consequently, all HIV-positive children under the age of 14 years who enroll at service points should receive nutritional packages consisting of vitamin syrup and a supplement meal" (South Africa Department of Health 2003, p.42). It is under this heading that the issues of caregivers and child-headed households as well as the need for "appropriate" counseling are referred to for the first time. However, this reference to counseling alludes only to nutritional management and neither antiretroviral treatment literacy nor medicine administration strategies are mentioned.

\section{Continuum of Care Services}

The operational plan recognizes that "over the course of diagnosis and care for HIV and AIDS, numerous providers and delivery systems interface to address patient needs" (South Africa Department of Health 2003, p.29). It recommends the "designation of a care coordinator to maximize coordination of patient services, including linkages with adherence and ancillary support systems, referrals, and follow-ups with diagnostic and consultant services at other locations..." (South Africa Department of Health 2003, p.29). The nature of childhood conditions and diseases lends itself to frequent up-and-down referral between the various health service levels and services. It may therefore be even more critical in the case of pediatric HIV care and treatment to have a care coordinator. However, the plan does not explain how to operationalize this principle and the challenge for pediatric patients in the South African public health service context remains, since there is no universal access to specialist pediatricians in the public sector who may act as a care coordinator for children.

\section{Accreditation of Service Points ${ }^{1}$}

Because there are no explicit accreditation criteria for pediatric ARV treatment service points in the plan, it is therefore assumed that the criteria are generic and do not exclude pediatric sites (South Africa Department of Health 2003, pp.95-101). However, in the case of pediatrics, criteria number) regarding "availability of a trained care team on-site with representation of all relevant professions (clinicians, nurses and counselors)," may be difficult to achieve since not all clinicians are proficient in pediatric management. In addition, most service points familiar with the care of young children, particularly primary care clinics (EPI, IMCI) are nurse-driven services. To date, this has been a major barrier to delivering ARV treatment for children less than six years of age.

\section{Projected Treatment and Monitoring Costs}

The cost of HIV testing in infants less than 18 months of age is explicitly calculated in the operational plan, based on the Actuarial Society of South Africa's ASSA 2000 model's one year age-band projections. The conclusion reached is that this cost is a mere fraction (less than 1 percent) of the overall cost of laboratory monitoring: "Thus even if larger numbers of infants presented for care in the early

\footnotetext{
${ }^{1}$ The National Health Department accredited 122 sites by March 2005 of which 113 were operational. This process has taken longer than anticipated and the function of accreditation has now been devolved to the Provinces (HST 2005).
} 
stages of the program, these costs would easily be accommodated within the overall amounts budgeted for laboratory monitoring" (South Africa Department of Health 2003, p.249). This projection has not, however, taken into account other diagnostic monitoring such as CD4 counts and viral load testing for screening of children in need of ARV treatment.

\section{Planning for and Monitoring the Pediatric ARV Rollout}

Although it is explicitly stated in WHO's 3 x 5 target that approximately 10 to 15 percent of the total should be comprised of children on treatment (WHO 2003), the projected number of patients on treatment in the operational plan does not distinguish between children younger than 14 years of age and others. Table 1 illustrates the estimated number of children expected to be on ARV treatment by the end of March 2005 assuming that 10 and 15 percent, respectively, of the projected number of cases in the operational plan (p. 248) will be children under the age of $14 .^{2}$

Table 1 Expected total number of pediatric cases on ARVs by province

\begin{tabular}{|c|c|c|c|}
\hline Provinces & $\begin{array}{l}2004 / 05 \\
\text { adults }\end{array}$ & $\begin{array}{c}10 \% \text { estimate } \\
\text { children } \\
<14 \text { yrs }\end{array}$ & $\begin{array}{c}15 \% \text { estimate } \\
\text { children } \\
<14 \text { yrs }\end{array}$ \\
\hline Eastern Cape & 15,626 & 1,562 & 2,343 \\
\hline Free State & 11,883 & 1,188 & 1,782 \\
\hline Gauteng & 45,000 & 4,500 & 6,750 \\
\hline KwaZulu-Natal & 74,208 & 7,420 & 11,131 \\
\hline Limpopo & 21,494 & 2,149 & 3,224 \\
\hline Mpumalanga & 10,767 & 1,076 & 1,615 \\
\hline Northern Cape & 2,492 & 249 & 373 \\
\hline North West & 10,426 & 1,042 & 1,563 \\
\hline Western Cape & 5,728 & 572 & 859 \\
\hline Total in SA & 197,624 & 19,762 & 29,643 \\
\hline
\end{tabular}

Table 1 indicates that the total number of children under 14 years of age who should be on treatment in South Africa by 2005 is approximately 20,000 at the minimum and 30,000 at the 15 percent estimate. The Western Cape exceeded its target in March 2005 with 1,284 children reported to be on treatment in the Province (Provincial Health Department of the Western Cape 2005).

\footnotetext{
${ }^{2}$ The figures in the operational plan are based on the ASSA 2000 model.
} 
There is very little data for accurate estimation of children in need of ARVs, and no strategy has been implemented by the National Health Department to ensure the accurate reporting of the numbers of children on treatment under the age of 14 years. For example, under the primary set of indicators to be reported within a 6-month period, the only reference to children is the variable "proportion of registered patients on regimen 1a, 1b, 2 or child regimen." This clearly excludes instances where adult regimens (pills) are given to children and may thus lead to an underestimation of the number of children on treatment if extrapolations are made from such aggregated data. Clearly, this lack of accurate monitoring of children in need of treatment will have a ripple effect on all service provision planning.

\section{Conclusion}

This review indicates that while children are assumed to be part of the target population for the ARV rollout in South Africa, there is insufficient attention to child-specific aspects of monitoring and evaluation of the ARV program and subsequently, there are fewer of the resources required to facilitate access to treatment for children less than 14 years of age.

Access to ARVs hinges on disease staging using laboratory and clinical indicators, which is impeding the implementation of the rollout of the pediatric ARV program. The current practice of taking blood from veins for CD4 counts and viral load testing poses a particular challenge in young children and infants because of the lack of trained personnel willing to do this task. To overcome this challenge, a concerted strategy to train and encourage nursing staff to do phlebotomy procedures on young children should be implemented nationally.

The South African government's policy and intent regarding the management of HIV-infected children can be contextualized in yet another policy document entitled "Policy framework for non-communicable chronic conditions in children (South Africa Department of Health 2002)." Although this document focuses on noncommunicable diseases, it comprehensively describes the management of chronic conditions in children in South Africa. Key issues applicable to long-term chronic care are listed in the document, including that chronic conditions are lifelong and often incurable, that the conditions require multi-drug regimens, that lifestyle adjustments will be needed, and that the condition can be controlled. Chronic care should be underpinned by comprehensive integrated services, effective communication between providers and consumers, referral systems that promote continuity of care, strong community links and support, and a caring ethos. Furthermore the roles, responsibilities, and services at primary level clinics, community health centers, district hospitals, regional hospitals, and tertiary hospitals should be clearly defined and supported by appropriate training and technical expertise. Consideration is given to the creation of an efficient referral system, drug procurement and supply, and the transition from child- to adult-oriented care. This document provides a complete framework for the management of chronic conditions and could serve as a starting point for revising the policy recommendations for managing HIVinfected children in South Africa. 


\section{Overview of Methods and Study Sites}

\section{Methods}

Pediatricians in the country who were providing antiretroviral treatment to their patients were asked to participate in the study and invited to a consultative workshop with stakeholders in Johannesburg in January 2005 (Horizons and University of Cape Town 2005). Participants in the planning meeting had the opportunity to offer input into the questionnaire design, protocol refinement, and site identification. Managers of identified sites were contacted (in many cases this was the person who participated in the planning meeting), and those who agreed to participate and obtained the necessary formal permissions from health and institutional authorities were included in the site visits. As can be seen in Table 2, 16 study sites in 5 provinces were visited for the situational analysis, 11 of which had been identified during the consultative workshop. The rest were identified through snowball sampling. ${ }^{3}$ Public and NGO health facilities at tertiary, secondary, and primary levels were included in the study.

The situational analysis utilized qualitative and quantitative methods to collect data at the ARV program sites, namely, facility observations and semi-structured questionnaires administered to facility managers, health care workers, and caregivers. The semi-structured questionnaires were adapted for the various categories of health care workers, i.e., doctors, nurses, pharmacists, social workers, and counselors. Openended interviews were conducted with provincial managers and dieticians. Structured questionnaires were administered to a convenience sample of caregivers of children on antiretroviral therapy attending clinics during the periods of the field visits.

The protocol was reviewed and approved by the Institutional Review Board of the Population Council, the Research Ethics Committee of the University of Cape Town, and the Human Research Ethics Committee (Medical) of the University of the Witwatersrand. Permission to conduct the study at the sites was obtained from the provincial health departments and facility managers.

The researchers included three trained HIV counselors, one of whom was multilingual (English, Afrikaans, Xhosa, Zulu, Sotho, Tswana), who administered questionnaires to caregivers of children on ART and interviewed counselors at the sites. Two medical doctors and one registered professional nurse conducted interviews with health facility managers and other health care workers. The interviewers took field notes during observations and recorded general descriptions of sites visited (see Appendix).

Interviews with health care workers were tape recorded with permission (two health care workers refused to be tape recorded but granted interviews). Each participant was informed of the purpose of the study and written consent was obtained. Each participant was offered a copy of the consent form.

The fieldwork commenced in the Free State Province on 11 April 2005 and ended in the Western Cape Province on 21 June 2005. All service statistics gathered from sites was for the period of inception of the pediatric ARV program through 31 March 2005.

\footnotetext{
${ }^{3}$ Snowball sampling is a technique commonly used in the behavioral sciences to identify study participants. It is based on the identification of an index participant fulfilling the study criteria and asking that participant for other leads.
} 
Table 2 Study sites

\begin{tabular}{ll}
\hline Province and site & Facility level \\
\hline Western Cape & \\
$\quad$ Red Cross Hospital, Cape Town & Tertiary hospital \\
Site B Clinic, Khayelitsha & Primary care clinic \\
Site C Clinic, Khayelitsha & Primary care clinic \\
Eastern Cape & \\
$\quad$ Cecilia Makawane Hospital, East London & Tertiary hospital \\
Frere Hospital ARV clinic, East London & \\
St Elizabeth's Hospital, Lusikisiki & District (secondary) hospital \\
Xorana Clinic, Lusikiski & Primary care clinic \\
Village Clinic, Lusikiski & Primary care clinic \\
Palmerton Clinic, Lusikiski & Primary care clinic \\
Goso Clinic, Lusikiski & Primary care clinic \\
Gauteng & \\
Harriet Shezi, Baragwaneth Hospital, Johannesburg & Tertiary hospital \\
Zola Clinic, Soweto & Primary care clinic \\
Kwa Zulu Natal & \\
McCord Hospital, Durban & NGo (secondary) facility \\
Free State & \\
National District Hospital, Bloemfontein & Tertiary hospital \\
Sunflower Home* & "Step-down" facility** \\
Munguang Community Partnership Program, Rocklands & Primary care clinic \\
\hline
\end{tabular}

* Situated on the premised of the National District Hospital

**Definition: A step-down facility is defined as one that provides care for patients who are not deemed "sick" enough for hospitalization but not 'well enough to be cared for at home by family members (i.e. a degree of nursing care is needed).

Someone other than the interviewer transcribed all tape-recorded interviews, which were then checked by the interviewer. The qualitative data was organized using EZ-text ${ }^{\circledR}$ (CDC's qualitative analysis software package), followed by manual coding of all themes. Coded quotations were listed according to themes using Microsoft Word ${ }^{\circledR}$ with reference to the transcript source. Interviewers took detailed notes of the two interviews with health care workers who refused to be tape-recorded; these were written up directly after the interview was conducted and incorporated into the analysis.

Data for the caregiver questionnaires were captured and analyzed using EPI INFO ${ }^{\circledR}$ (Version 3.3.2 February 2005) and SPSS. Individuals not involved in administering the caregiver survey conducted the capture, cleaning, and analysis of data.

The preliminary results of the study were presented to a meeting of site collaborators, representatives of the Department of Health, and other stakeholders on 22 September 2005. The purpose of the meeting was to provide an opportunity for the collaborators to provide insights into the interpretation of data and make recommendations regarding the study's final report. 


\section{Description of Sites}

Data on the characteristics of 7 of the 16 pediatric ARV services was collected from the facility managers $(\mathrm{n}=7)$, all of whom were medical doctors by profession. Facility managers were interviewed about staff composition, patient case loads and profiles, patient targets, physical capacity and infrastructure, staff, training and capacity, involvement in pediatric outreach services, partnerships and collaborations, treatment and drug policies/guidelines, availability of drug regimens, diagnosis and screening, clinical and virological monitoring, referral practice, patient tracking and adherence monitoring systems, data collection and monitoring tools, and perceptions of major challenges.

The sites surveyed varied by level of care (tertiary, district hospital, primary level clinics). Regardless of the type of facility, the initiation of the ARV services emerged mostly out of the vision, dedication, and research or clinical interests of a pediatrician or medical doctor at the site. All primary level sites were supported by tertiary or district hospitals for the initiation and management of children on ART. The exception was Médecins sans Frontières (MSF) clinics, where primary care doctors were initiating children on treatment and only referring complicated cases to the visiting pediatrician or to the tertiary hospitals.

These seven sites collectively reported a total of approximately 1,300 children on ARV treatment by the end of March 2005 (range 39-550) with approximately 60 percent children under the age of six years. The initiation dates for the pediatric ARV services at the participating sites ranged from March 1999 to July 2004. 
Table 3 Facility/site statistics as of March 2005

\begin{tabular}{lccc}
\hline Province/facility level & $\begin{array}{c}\text { HIV case load } \\
\text { (excluding those } \\
\text { on treatment) }\end{array}$ & $\begin{array}{c}\text { No. on } \\
\text { ARVs }\end{array}$ & $\begin{array}{c}\text { Proportion of } \\
\text { children < 6 yrs } \\
\text { (HIV/ARV clinic) }\end{array}$ \\
\hline $\begin{array}{l}\text { E.Cape 1 } \\
\text { Primary level_rural (4 clinics and district } \\
\text { hospital) }\end{array}$ & 105 & $39^{\star \star}$ & $50 \%$ \\
$\begin{array}{l}\text { E.Cape 2 } \\
\text { Tertiary level_rural town }\end{array}$ & Not available & Not known \\
$\begin{array}{l}\text { W.Cape 1 } \\
\text { Tertiary level (hospital site) }\end{array}$ & $300^{\star}$ & 351 & $85 \%$ \\
$\begin{array}{l}\text { W.Cape 2 } \\
\text { Primary level (3 clinics) }\end{array}$ & 248 & 157 & $68 \%$ \\
$\begin{array}{l}\text { Gauteng } \\
\text { Tertiary and primary level (hospital site and 1 } \\
\text { clinic) } \\
\text { KZN }\end{array}$ & & 550 & $60 \%$ \\
Secondary level (district hospital) \\
$\begin{array}{l}\text { Free State } \\
\text { Tertiary and primary level (hospital site and } \\
\text { referral clinic sites) }\end{array}$ \\
\hline
\end{tabular}

*Only if additional staff is provided to allow 75 patients a week to be seen and then 300 patients per month can be managed for constant follow-up.

**By the time of the field visit the numbers had risen to 53 .

\#"The ARV clinic does not have many children not on ARVs-ARV initiation is quick in patients able to cope...we are unable to provide the actual number HIV infected not on treatment." -Facility manager

Facility managers were asked, "How many children are you planning to initiate on treatment within the next financial year (April 2005-March 2006)?” The responses are shown in the final column of Table 4. Facility managers described various means of arriving at targets, which are shown in Table 4. 
Table 4 Projection of new patients on treatment for $2005 / 6$ per site

\begin{tabular}{llc}
\hline Province & \multicolumn{1}{c}{ Rationale } & \multicolumn{1}{c}{$\begin{array}{c}\text { Additional cases for } \\
\text { treatment 2005/6 }\end{array}$} \\
\hline $\begin{array}{l}\text { E. Cape 1 } \\
\text { Rural }\end{array}$ & Based on 10 percent of the HIV prevalence rate. & 50 \\
$\begin{array}{l}\text { E. Cape 2 } \\
\text { Rural Town }\end{array}$ & $\begin{array}{l}\text { Based on current capacity plus an additional doctor } \\
\text { and nurse team. }\end{array}$ & $\pm 160^{*}$ \\
W. Cape 1 & $\begin{array}{l}\text { Based on approximately 20 new patients per month. } \\
\text { But as many who need ARVs. There is no waiting list. }\end{array}$ & $240^{\star *}$ \\
W. Cape 2 & $\begin{array}{l}\text { Based on actual numbers of HIV positive births. But as } \\
\text { many who need ARVS. There is no waiting list. }\end{array}$ & $220^{* *}$ \\
Gauteng & $\begin{array}{l}\text { Based on WHO 3 x 5 estimates of 10 percent of adult } \\
\text { patients. }\end{array}$ & 240 \\
KZN & $\begin{array}{l}\text { A total of 20 new patients per month of which 20 } \\
\text { percent are estimated to be children. There is no }\end{array}$ & \\
waiting list. & $\begin{array}{l}\text { Double the existing numbers due to expansion of } \\
\text { pharmacy. }\end{array}$
\end{tabular}

*Only if additional staff is provided to allow 75 patients a week to be seen and then 300 patients per month can be managed for constant follow-up.

**Target number, but will accept more pediatric patients if necessary.

All seven facilities had dedicated days for ARV clinic appointments (these days governed the timing of the research visits in order to target caregivers of children on ART). Most sites allocated one or two sessions to ARV clinics per week. Integrated adult and pediatric sites tended to see adults and children on the same day (Table 5). 
Table 5 Clinic days for pediatrics per study site ( $\mathrm{Y}=$ yes, $\mathrm{N}=$ no)

\begin{tabular}{lccccc}
\hline Province & \multicolumn{4}{c}{ Pediatric clinic days per site } \\
& Mon & Tues & Wed & Thurs & Fri \\
\hline Free State & $\mathrm{Y}$ & $\mathrm{N}$ & $\mathrm{Y}$ & $\mathrm{N}$ & $\mathrm{N}$ \\
Eastern Cape 1 & $\mathrm{Y}$ & $\mathrm{Y}$ & $\mathrm{Y}$ & $\mathrm{Y}$ & $\mathrm{N}$ \\
Western Cape 1 & $\mathrm{N}$ & $\mathrm{Y}$ & $\mathrm{N}$ & $\mathrm{Y}$ & $\mathrm{Y}$ \\
Eastern Cape 2 & $\mathrm{N}$ & $\mathrm{N}$ & $\mathrm{Y}$ & $\mathrm{Y}$ & $\mathrm{N}$ \\
Gauteng & $\mathrm{Y}$ & $\mathrm{Y}$ & $\mathrm{N}$ & $\mathrm{N}$ & $\mathrm{Y}$ \\
KwaZulu Natal & $\mathrm{Y}$ & $\mathrm{N}$ & $\mathrm{N}$ & $\mathrm{Y}$ & $\mathrm{Y}$ \\
Western Cape 2 & $\mathrm{Y}$ & $\mathrm{Y}$ & $\mathrm{Y}$ & $\mathrm{Y}$ & $\mathrm{Y}$ \\
\hline
\end{tabular}

The composition of staff (health teams) among sites visited range from a three-member team of a doctor, nurse, and pharmacist to multidisciplinary teams consisting of approximately 20 members including social workers, counselors, clerks, and dieticians.

Only two sites, Free State and Gauteng, reported having waiting lists of children to be initiated on ARVs. According to these sites, this was due mainly to the process of initiating treatment, that is, the time lag between identification, preparation, and initiation (Gauteng) and a lack of adequate staff (Free State).

\section{Health Provider Surveys and I nterviews}

Seventy-four interviews representing the 16 sites were conducted with various categories of health care workers, including the ARV facility managers, and 127 interviews were conducted with caregivers of children on ART. Medical doctors comprised almost half (45 percent) of the health care worker respondents, followed by nurses (22 percent) (Table 6 ). All facility managers were qualified medical doctors and all were instrumental in setting up the ARV clinics for children. 
Table 6 Summary of numbers of participants per province

\begin{tabular}{|c|c|c|c|c|c|c|c|}
\hline Category & Free State & E. Cape & W. Cape & Gauteng & KZN & Total & $\%$ \\
\hline $\begin{array}{l}\text { ARV facility } \\
\text { manager* }\end{array}$ & 1 & 2 & 2 & 1 & 1 & 7 & 9 \\
\hline Doctor & 4 & 3 & 11 & 4 & 3 & 25 & 34 \\
\hline Total doctor* & 5 & 5 & 13 & 5 & 4 & 32 & 43 \\
\hline Nurse & 2 & 6 & 4 & 2 & 2 & 16 & 22 \\
\hline Social worker & 1 & 1 & 1 & 1 & 1 & 5 & 7 \\
\hline Pharmacist & 1 & 2 & 1 & 1 & 1 & 6 & 8 \\
\hline Counselor & 0 & 3 & 2 & $3^{\star \star}$ & $4^{\star \star \star}$ & 12 & 16 \\
\hline Nutritionist & 0 & 0 & 0 & 1 & 0 & 1 & 1 \\
\hline $\begin{array}{l}\text { Provincial DOH } \\
\text { representative }\end{array}$ & 1 & 1 & 0 & 0 & 0 & 2 & 3 \\
\hline Total HCWs & $10(14 \%)$ & $18(24 \%)$ & $21(28 \%)$ & $13(18 \%)$ & $12(16 \%)$ & 74 & 100 \\
\hline Total caregivers & $4(3 \%)$ & $35(27.5 \%)$ & $38(30 \%)$ & $35(27.5 \%)$ & $15(12 \%)$ & 127 & 100 \\
\hline
\end{tabular}

*All ARV facility managers were medical doctors working at the pediatric ARV clinics.

${ }^{* *}$ These counselors were all based at one site (tertiary/research facility)

***These counselors were based at a NGO facility

Experience working in an HIV/ARV pediatric clinic ranged from 1 to 48 months (median 12 months) among those surveyed, while the years of experience as a qualified medical doctor ranged from 3 to 31 years (median 8 years).

Work experience as a nurse ranged from 3 to 27 years among respondents (median 15 years). Experience in HIV/ARV clinics ranged from 7 to 36 months (median 24 months). Table 7 illustrates the numbers of respondents per nursing category. It should be noted that the nurses surveyed were not a homogenous group and have varying scopes of practice.

Table 7 Types of nurses working in HIVIARV clinics

\begin{tabular}{lc}
\hline Nurse category & No. of interviewees in each category \\
\hline Chief professional nurse & 4 \\
Clinical nurse practitioner & 1 \\
Professional nurse & 6 \\
Enrolled nurse & 4 \\
Staff nurse & 1 \\
Senior assistant nurse & 1 \\
\hline
\end{tabular}

A total of 12 counselors were interviewed across the sites. No counselors were interviewed in the Free State, where they were predominantly based at the adult ARV clinic and the hospital to provide VCT services. The years of experience as a counselor in any counseling field ranged from 10 months to 14 
years, while experience in specific HIV counseling ranged from 10 months to 3 years. The duration of time working at the facility correlated closely with their HIV-specific counseling experience, namely, four months to three years. All but one of the counselors were female, and their employment status was split between volunteers, part-time hires, and full-time hires. Counselors were interviewed about their relevant pediatric-related HIV training and experience as well as their perspective on the main challenges facing the pediatric ARV rollout.

One social worker was interviewed in each province but this position only existed at the tertiary sites and the NGO health facility. Of the five social workers interviewed, three spoke Xhosa as their first language, one spoke Sotho, and one spoke Zulu. They were all qualified social workers whose highest formal education was a four-year social work degree. Four of the respondents were employed as full-time staff and one was part-time. However, none of the social workers was working specifically in the HIV/ARV service on a full-time basis.

Six pharmacists and one pharmacy assistant were interviewed. The pharmacists' work experience ranged from 3 to 20 years with an average of 6 years since qualification. The length of service at the facility ranged from 4 to 48 months. ARV dispensing experience ranged from 4 to 18 months with an average of 8 months. Pharmacists were interviewed about their relevant ARV dispensing experience, their main roles and responsibilities regarding ARVs, issues relating to drug procurement, storage and distribution of ARVs, dispensing of concomitant therapies (e.g., for TB), and their perceptions of the major challenges facing pharmacists with regard to the pediatric ARV rollout. Only three of the seven respondents reported that the ARV pharmacy was incorporated into the main pharmacy at the facility. 


\section{Health Care Worker Training, Experience, and Roles in Pediatric ARV Service}

\section{Doctors}

\section{Training and experience}

Most doctors reported that they received no formal training in the management of HIV in children. Instead, they mentioned "self-study" (i.e., reading books and on-line information); attending relevant conferences, seminars, and workshops such as those offered by the Department of Health on the ARV rollout and by academic institutions (which focused primarily on adults); as well as "mentorship training" from an experienced doctor. Below are some comments from doctors on their training and preparation for the ARV rollout for children, which illustrate a wide range of experience. Very few specifically mentioned receiving specific pediatric training:

"Last year in June [2004] the government was preparing to start the rollout, so all the doctors that are working in HIV clinics received training then from there, I just read books on the subjects...."

Doctor, primary care level in urban setting

"We had weekly sessions with the MSF doctors and they provided training with regard to HIV and its management and I had exposure to HIV management as a medical registrar in [a public hospital] for about 18 months in 2001/2."

Doctor, primary care level in rural setting

"I did the on-line course by Steve Andrews, that's the only training I did and just on-line on the Internet, otherwise I didn't have formal training, only mentorship for a very short time...2 weeks...."

Doctor, primary care level in rural setting

“Nothing...self-trained...."

Doctor, tertiary care level in peri-urban setting

"Previously I was at a provincial hospital so I received training as part of the ARV rollout training. Then I attended some of the HIV road shows and training that the Department of Health had in 2002. Last week I attended the Elizabeth Glaser-funded training for three days at the University...."

Doctor, primary care level in urban setting.

\section{Roles in ARV clinics}

Most HIV/ARV clinics are run on a session basis, such as on a designated day or certain hours every week. Therefore all doctors work in the ARV clinics part-time as part of their full-time positions at the clinic, which include several other duties and responsibilities ranging from specialist pediatric consultant for a district to hospital surgeon. 


\section{Clinical duties}

All doctors surveyed fulfilled clinical duties in the ARV program. This involved evaluating children for initiation of ARV treatment, monitoring, conducting follow-up consultations, and making referrals to auxiliary services or facilities. However, in the absence of support staff, such as counselors or nurses, some doctors also counseled patients, drew blood, and assessed patient adherence to medication.

Those who were specialist pediatricians tended to see children with more complicated opportunistic infections or acute serious conditions. Some doctors did not specialize in either children or adults and saw both.

\section{Training and mentorship}

Experienced doctors or specialist pediatricians tended to fulfill a training and mentorship role to their colleagues (on-the-job training). Some were actively involved in strengthening or setting up pediatric ARV services at peripheral sites (outreach services).

In addition, some doctors at tertiary institutions were volunteering their services at the HIV/ARV clinics because they were not employed by the province but by a research project and therefore primarily fulfill a research role; others filled an academic role.

\section{Supervision and management of clinics}

Doctors involved in pediatric ARV services also had supervisory and management functions at the facilities and some were designated "ARV coordinator." Their management functions included managing staffing for the clinic as well as related issues, including ensuring procurement of supplies and equipment.

\section{Nurses}

\section{Training and experience}

About one half (9/17) of nurses reported attendance at 3- to 10-day training workshops on ARVs offered by MSF, AIDS Training Information and Counseling Centers (ATICC), or overseas consultants "from America." Five nurses reported that they had undergone the government rollout training. Some nurses (4/17) reported that they had completed counseling courses (e.g., VCT courses) in relation to the PMTCT training given in districts. Other respondents (3/17) reported enrollment in the University of South Africa HIV Management Certificate course. In addition, nurses mentioned mentorship training by doctors at the facilities as a primary source of training related to pediatric HIV management. 


\section{Roles in ARV clinics}

Nurses' roles in the HIV/ARV clinics included weighing and measuring children, taking histories, ensuring that patients are aware of their follow-up appointment dates, and following up with patients who missed their appointments. Others reported that they were involved in the management of children including phlebotomy, pre-screening for ARVs, and preparing them for treatment, after which a doctor prescribed treatment. Nurses conducted follow-ups of patients on treatment, including monitoring nutritional status, drug adherence, and side effects.

Some reported holding a child while the doctor took blood, treating common ailments like diarrhea, and giving Bactrim to children born to HIV-positive mothers. However, many reported that this is only done under a doctor's supervision, especially in the case of very young children:

"...I rehydrate them, we advise them about diet...then we prescribe some medication to those who didn't see the doctor-the Bactrim, folic acids—although we always want the doctor to intervene."

Nurse, primary care level in rural setting

Several nurses expressed a preference for working with children over the age of eight years. They shared the perception that treating young children and prescribing ARVs to children is complicated due to the calculations used for dosing.

"I treat opportunistic infections and manage adults on ARVs. I can do the children if they are above eight years...below that age they're on syrups...syrups are a bit complicated and they need a more professional person to do it."

Nurse, primary care level in peri-urban setting

Most nurses surveyed were predominantly involved in counseling adult patients and caregivers of pediatric patients and providing drug readiness and HIV education. None reported counseling children directly.

"...counseling the caregivers of children. Finding out their problems at home about managing the children...also assisting those who are unable to get food for the children by arranging with the social worker to assist with food or grants. I'm also assisting the pharmacist in checking consistency [adherence counseling] with the mothers about treatment and about any problems that they encounter with the treatment.

Nurse, tertiary care level in peri-urban setting

Some nurses reported having to "sit in" with the doctors as they consult with patients to assist as interpreters and to check adherence to medication, while learning about HIV and treatment management at the same time.

"I act as an interpreter for the doctors and patient when they are having the problems with expressing themselves - to make the patient understand what they [the doctors] want them to dobecause most of the doctors they are the white doctors."

Nurse, primary care level in urban setting 
“...we are not giving ARVs yet, it is only the doctor who issues ARVs, but we are usually sitting next to the doctor to learn...."

Nurse, primary care level in rural setting

Several nurses reported that they performed data entry or data management of patient records. It was evident that the patients in the HIV/ARV clinic were being monitored differently than other patients attending the service, which required a system that would facilitate patient tracking.

"I manage (among other duties) the patient database and the monthly provincial statistical reports."

Nurse, tertiary care level in urban setting “...we also do patient statistics...we're only concerned with the patients who are on treatment and the number of patients we treat daily...we record it in a file. We also complete the required statistical forms and ordering of stock."

Nurse, primary care level in urban setting

Perhaps an unanticipated extra administrative task emerging out of the pediatric ARV clinic was the need to capture data that did not conform to existing formats, hence the emergence of a duplicate folder system in some settings as part of a larger health service such as a tertiary center.

“...I see that all the procedures are in order for the doctor...most of the patients coming into the clinic now are on treatment; they also have a different folder. I have to see that the folder is in the original folder and the data sheet for the doctor is in..."

Nurse, tertiary care in urban setting

At the academic hospitals nurses' roles included research activities, thus they were more likely to be involved in phlebotomy and nursing management; this involved ensuring adequate stock levels, staffing, supervision, and patient flow.

Based on interviews with nurses, it is apparent that many of them mainly saw adults and then "translated" the care to children when they needed to see them. In other words, the service delivery was not specifically devised for children; instead, the nurses adapted adult procedures.

\section{Counselors}

\section{Training and experience}

Most counselors had undergone basic counseling training through one of these institutions: Lifeline, ATICC, UNISA, Department of Health, MSF, or UNICEF/HARVARD Training Collaboration (NATAL).

Those with more experience tended to have been trained in basic counseling, PMTCT, VCT, and homebased care training, while those who entered the field more recently received specific HIV and adherence counseling. However, this training was focused on the counseling of adults. 


\section{Roles in ARV clinics}

The counselors viewed their role in the HIV care team as being instrumental in ensuring that those who start on ARV treatment continue to stay on it. They believed that they were ideally placed to identify potential barriers to adherence and that they had the ability to encourage and support patients to take their medication.

"...the ARVs are not about the doctor and the pill, it is about my ability to take it, make a habit of

it...now that you have the drugs—drink it! But that isn't typically motivated by a physician-it's

typically the counselor that identifies the barriers to taking the drugs and can encourage the person best to take it most adherently."

Counselor, secondary level hospital

\section{Social Workers}

\section{Training and experience}

Two of the five respondents had no experience in HIV-related work (such as counseling and support of HIV-infected and affected persons) while the remaining three had one, three, and eight years experience, respectively.

The majority received no specific ARV-related training and no pediatric-specific training. Three of the respondents attended the ATTIC HIV training, which included an adolescent care and training course, a peer counseling training course, and a pre- and post-test counseling course. Only one social worker reported having attended the ARV rollout training workshops offered by the Department of Health. One social worker reported having taken an AIDS-related bereavement course aimed at social workers.

\section{Roles in ARV clinics}

The social workers interviewed described their various roles in relation to the HIV clinic as supporting and supervising counselors, accessing social support for the patients (processing grant applications), counseling patients who have experienced rape, and providing treatment education to patients (need for adherence). In addition, they coordinated the follow-up of patients at home.

\section{Pharmacists}

\section{Training and experience}

All pharmacists employed in the public sector have a degree in pharmacy. The pharmacists' experience since qualification ranged from 3 to 20 years (average of 6 years). The length of service at the facility ranged from 4 to 48 months, while the length of experience in ARV dispensing ranged from 4 to 18 months (average 8 months). 


\section{Roles in ARV clinics}

The usual dispensing duties and patient counseling role of pharmacists has been augmented due to the nature of ART and the risks of drug resistance. Pharmacists now spend more time packing, labeling, color-coding bottles for easy recognition, and color-coding medication administration tools like syringes to facilitate ease of measurement. At one site all pediatric syrups were decanted into $200 \mathrm{ml}$ bottles, with the rationale that the medicine caps are difficult to open and that the amount of medicine containers carried out by the patient could be reduced. This decanting practice was very time consuming and not very efficient. However, it was done in an attempt to ease the stigmatization of patients who have to use public transportation while carrying a load of identifiable ARV medication.

Pharmacists spent considerable time explaining to patients how important it is to take ARVs correctly and ensuring that they understand the dosing requirements. ARV drug procurement and stock control was another substantial task that pharmacists in most sites were responsible for overseeing.

Pediatric formulations are challenging because the dosing requirements change as the child grows and caregivers need to be re-trained with regard to dosing at almost every visit, especially for a young child.

"You must emphasize it more—really make sure that they are using it correctly."

"Adherence and understanding required by the mother is more complex."

Pharmacist, district hospital setting

Pharmacist, tertiary care level in metropole setting

As a result, pharmacists found that supporting and assessing adherence in children was difficult.

"...you actually have no way of telling and seeing if the mom is taking the exact quantity or giving the child the exact quantity."

Pharmacist, tertiary care level in metropole setting

An additional task resulting from dispensing ARVs was adherence monitoring. Some pharmacists were engaged in actual pill counts and measuring returned syrups. The pharmacists also had to assess whether medication storage was adequate at home.

“...checking returned meds to monitor adherence by counting tablets and measuring syrup. Checking doses prior to giving to patients and dealing with returns...calculate next appointment date and give an extra two days meds...It takes approximately 30 minutes for one folder to be processed and going through problems with the mother."

Pharmacist, tertiary care level in metropole setting

“...find out if there are any problems at home and how medicine is stored. Check if they can understand and explain how the medication is given by demonstrating syringe use. Check with the mother if she understands dose changes and reconfirm the change [if any]...."

Pharmacist, tertiary care level in metropole setting

Pharmacists worked closely with any HIV counselors available on site. This reliance on counselors was often due to the language barrier that exists between many pharmacists and their patients. 
"...if we find the patient does not understand, we refer them to a counselor who can speak to them in their language and make sure the patient knows...."

Pharmacist, secondary care level in urban setting

Other roles and functions reported were organization of support groups and income generation for HIVinfected patients, ARV outreach programs to clinics, and other non-ARV related pharmacy duties and dispensing.

“...I'm part of trying to get the support group running and trying to start the income generation for them."

Pharmacist, tertiary care level in urban setting 


\section{Current and Optimal Sources of Referral for Children to ARV Treatment}

\section{Current Sources of Referral to ARV Clinics}

The findings indicate that the majority of children were referred by (1) other health facilities, especially primary care facilities where no ARVs were being offered (there were instances of up- and-down referrals along the levels of care, i.e., from clinic to tertiary and back down); (2) in-patient wards at tertiary hospitals; and (3) "self-referrals," which occurred among HIV-infected patients on treatment who then brought their children in for testing and care.

"Most of our patients come from mothers who are already at our clinic...."

Doctor, district hospital in rural setting

“...especially parents that are on treatment or know that they are HIV-positive will bring their children in for testing....”

Doctor, primary care level in peri-urban setting

“...the majority are referred from within the hospital...."

Doctor, tertiary care level in metropole setting

In the Free State, the ARV service was comprised of one treatment facility (which initiates therapy and monitors the patients) and several "assessment sites" where patients were diagnosed and staged ${ }^{4}$ before referral to the treatment site. In the rest of the provinces, ARV sites surveyed tended to provide HIV diagnosis, staging, and treatment at one facility.

Reports of referrals from private doctors and PMTCT programs were rare among the sites surveyed. There was, however, a trend where children's homes and non-governmental institutions caring for children infected with HIV were bringing them to health facilities for HIV care and treatment.

\section{Optimal sources of referral}

Health workers agreed that PMTCT, primary care clinics, and VCT services were the optimal sources of referral for the majority of children, as this would help children at risk to be identified early.

“...optimally it should be early identification and referral of patients before they get sick... unfortunately that's not the nature of most children referred to us ...[due to] the ineffective plan in the care for HIV [infected children] until recently... and so we're dealing with a huge back log and so tend to focus on the really extreme end of the spectrum which is not ideal...."

Doctor, tertiary care level in urban setting

\footnotetext{
4 "Staging" refers to the application of clinical and virological criteria to determine the stage of HIV progression.
} 
Doctors at tertiary institutions stressed that primary level clinics should be the place where children are identified, assessed, and treated, and that their only contact with the tertiary institution should be for complications and hospitalization. They added that referrals should come from the in-patient wards to their clinics, which are run on an outpatient basis.

“...well I think [our] role in the program is to support, mentor, and develop capacity of primary sites when it comes to the management of children infected and those requiring ARVs. [Our] specific clinic function will come to the management of more complicated cases, where patients are ill, or for the very young children under six months but certainly [tertiary institutions] are not appropriate for the management of children who are well and stable on ARVs...I mean if any person at a primary level of care is not able to manage that particular child for various reasons, then that's a time for referral to us otherwise, no."

Doctor, tertiary care level in urban setting

"We would ideally like to source referrals from infants and children who've already undergone a process of clinical staging and immunological staging, so they've got clinical stage and CD4 count and...been assessed to require treatment. We would welcome referrals of patients who have complicated illnesses...children who are just too unwell to be followed up in a primary center, or come from an area where there just isn't that service...Ideally, treatment of these children will actually be initiated at a community site, at a regional site, and even at a primary site, and we would see the children that develop problems on treatment - either they're failing treatment, they've developed complications of the treatment...."

Doctor, tertiary care level in urban setting

The perception was that there is a breakdown in follow-up of the exposed child between the time when the mother delivers and when other services are rendered at another health facility, such as primary care clinics for immunization follow-up.

"If things are running very well, the PMTCT program would have been the perfect point to refer the kids to us, but ja, it's not really running properly..."

Doctor, primary care level in rural setting

"We know there are children who should have been on ARVs from PMTCT, so we are grossly undersupplying ARVs to children and our PMTCT program is not working at this site and as a result children are dying in hoards! Our mortality has risen from about 1.5 percent of all non-admitted newborns and probably around 4 to 5 percent of all admitted children in 1994-5 who died [of causes related to HIV] to 48 percent of all deaths in 2000 due to HIV (and that's only the ones that we have confirmation of status or confident of the diagnosis). We are sure that it's much worse now five years down the line. Unfortunately we are not able to supply that data because our system is broken."

Doctor, tertiary care level in peri-urban setting

However, additional skills, such as blood taking from infants, are needed at the primary level for a complete assessment of children to determine the need for treatment. Beyond that there are logistical issues of CD4 count and viral load tests, which require laboratory services and reliable transportation services. While recognizing that primary care is most accessible to the patient and the community, health providers recognized the lack of capacity at that level to fulfill the range of services required to provide ARVs to children at present. 
“...we're trying to get the primary health care nurses to refer PCR-positive infants before they're clinically unwell because they can't do CD4s in primary care at the moment, so we are trying to get them to refer right at the moment of diagnosis and then we can assess the infants. So that's starting and I think it's going okay-we're seeing more, since we've got the 14 week PCR and we're seeing more and more very young infants...."

Doctor, primary care level in peri-urban setting

The historical evolution of the pediatric ARV rollout influences the way services are currently provided in the country. As one doctor described, it is the result of the way resources were allocated from research activities.

“...the nature of the pediatric ARV rollout is that it's been centered at teaching and tertiary centers and we're now trying to move it out peripherally rather than the other way round...."

Doctor, tertiary care level in urban setting

Another suggestion for optimal sources of referral is identifying children through the VCT services currently provided for adults, to find children who may have been missed by PMTCT services.

"...staff at the day hospital currently provide VCT services to adults and refer those testing positive to the HIV clinic. They could include the testing of children that come through their services in their VCT service and likewise refer those testing positive and who may have fallen through the cracks in the system."

Doctor, primary care level in rural setting 


\section{Major Challenges to Pediatric ARV Rollout}

Health providers were asked what they thought were the major challenges to pediatric ARV rollout at their facility. Lack of staff, poor infrastructure, policy and procedures, socioeconomic conditions, and, to a lesser extent, funding dominated the responses. Several strong opinions regarding how these issues may be addressed emerged.

\section{Human Resource Challenges}

All participants surveyed unanimously echoed the fact that human resources are the most critical component of the ARV rollout. It was evident through fieldworker observations that with trained, confident, and dedicated staff, much can be accomplished with very few resources.

\section{Lack of staff}

Everyone agreed that more personnel capable of treating children were needed. The lack of staff, including nurses, pharmacists, and auxiliary staff such as social workers and counselors, and staff capacity were most commonly cited as the major obstacles to increasing access to treatment for HIVinfected children.

"...there are hundreds of children who need to be on the program who are not because we don't have staff. So we've not removed treatment from anyone we've started but we've failed to start treatment on hundreds of them we ought to have...I think the biggest challenge is that the number of children who require ARVs far outnumber the resources available to provide that and the resource of greatest constraint is going to be human resources...doctors, nurses, and pharmacists. Employing volunteer staff is not going to resolve this...."

Doctor, primary care level in urban setting

“...we don't have the support staff such as counselors, social workers. In terms of language, counseling is a huge problem-there's not that sort of support staff such as the counselors, community workers, nurses. This seriously hampers our ability to rollout. There's also not sufficient medical staff here."

Doctor, tertiary care level in urban setting

"All the clinics we are working at we know that they are understaffed, they have a number of activities [to fulfill] - the gap is mainly professional nurses-it would have been ideal to have one nurse assigned only to deal with HIV patients.”

Doctor, primary care level in rural area

Most of the pediatric HIV/ARV services only had limited sessions per week (on one or two days in most settings). Increasing the number of sessions per week to accommodate the demand for care is not a feasible alternative in resource-constrained settings, because staff members serving in the HIV/ARV clinics have other general health service functions and may not be available at other times. 
“...the clinic is overflowing. He [doctor running the clinic] has to open more days but opening more days means another doctor is needed-but now the pharmacist cannot be there because the adult clinic is waiting for the same pharmacist you see. So you find that we say we must get out to the community but how will we do that without staff? It's a huge crisis and it's even difficult to plan how many patients you wish to start on treatment by a certain date because it puts a strain on the system....”

Doctor, tertiary care level in peri-urban setting

One of the respondents explained how they tried to overcome the challenge of increasing patient numbers per clinic by lengthening the follow-up period between patient visits. However, this system is limited because there is a point beyond which follow-up periods cannot be extended.

"...we are taking new children at the moment even though we have explained to management ....that we didn't have adequate staff to take them and the way we've done that is to in fact increase the period between follow-ups for children that are stable so we now see children every one and a half to two months and this has effectively doubled the number of children who are able to hold onto chronic follow-up. There remains the question of how long we can string out the follow-ups and it would be far more effective to have sufficient number of staff so... we can increase our efficiency...."

Doctor, primary care level in urban setting

Unfortunately, even when resources were allocated to augment the staff, certain provinces had difficulty getting suitable applicants and posts were often re-advertised and remained vacant due to a lack of applicants.

“...we need nurses, admin staff, and doctors. We advertised these posts and could not fill the doctor posts so we've had to re-advertise the positions [which in the meantime remain vacant]...."

Health facility manager, tertiary care level in rural setting

\section{Staff fears of treating children}

Health care workers reported a fear of treating children because they lacked training and experience in managing HIV infection in children, and the rollout training included very little information or reference to pediatric issues.

"...I think the doctors are quite insecure in their ability to give the ARVs to children. You treat children totally different to adults. This makes them nervous and a little bit unsure...in the rollout training there's very little pediatric training."

Doctor, primary care level in urban setting

"A lot of doctors are not willing to work with children and are scared of the rollout, they think that with children it's more difficult, so I know for instance our sisters [nurses] don't see children and some of the doctors don't see children."

Doctor, tertiary care level in urban setting

Some respondents suggested that while initial medical training should prepare doctors and nurses for caring for children with HIV infection, nurses should have more on-the-job training. 
"I think in the beginning, before we start working, the initial training should be made a little bit more [with respect to children]. The children are not just a sideline to the adults. I think you need at least two days of training just on the children. I must say that from my own experience, practice makes perfect and once you've been doing it a few times, you become quite confident...I think [nurses] need the same sort of thing...just to be made aware of the fact that you can treat children, it's not that difficult...The sisters are very scared, I think they're more scared of practical things like not getting blood out of a kid, but I think they would benefit from additional in-service training."

Doctor, primary care level in urban setting

A fieldworker wrote about an interview with a nursing manager at a district hospital, saying, "her nursing staff in the pediatric ward had also expressed their concern to her regarding their lack of ARV rollout training. They say they are left with feelings of insecurity and immense inadequacy... her criticism is leveled at the medical authorities for not heeding this matter as priority."

The dynamic evolution of the state of knowledge regarding HIV infection in children and the effects of ART on them results in constant change in treatment strategies. There is a need to constantly update medical personnel regarding these changes, especially those who do not have ready access to the internet.

"I think I do need it [additional skills/training to improve my ability to treat children on ARVs] because the knowledge of HIV in children is developing, new developments all the time, new drugs, new treatment all the time.... We just have to keep up with the changing information all the time...."

Doctor, primary care level in urban setting

\section{Nurses' reluctance to draw blood from children}

Nurses who are proficient at drawing blood from young children are critical to a pediatric ARV service. Generally, healthy infants do not need to have blood taken and therefore nurse-driven primary health care clinics have not needed to be proficient in phlebotomy for infants and children. The primary focus was previously on immunization, growth monitoring, and treatment of common childhood illnesses. However, the advent of the HIV epidemic has resulted in the need to diagnose and assess the stage of HIV infection and monitor toxicity of ARVs in children, which can only be done by testing blood.

Survey participants viewed the lack of blood-taking skills among nurses as a major barrier to the rollout of ARV treatment of infants and children. It is time-consuming and hazardous to health care workers to take blood from often unwilling children who have to be coaxed into allowing the procedure and may thrash about, needing to be held down.

A fieldworker's observation from a tertiary hospital site reiterated this barrier: "Currently nurses are refusing to take bloods on all children $<6$ years which impacts negatively on the screening process for ARV readiness. The children now have to be screened by the doctors in the ARV clinic, sent back to the fully booked drug readiness program, and then back to the ARV clinic for their ARV treatment to be initiated." 


\section{Emotionally difficult work}

A recurring theme among counselors was that their work was emotionally and physically demanding because they are constantly giving "bad news" (disclosing a positive diagnosis) and because workloads are heavy.

"...sometimes you find that we are so tired and the patients come to us with 5 minutes left before going home time-there's too many patients."

Counselor, tertiary care level in urban setting

Other health care providers echoed these sentiments about the emotional investment their work requires.

"My patients are special-you get a bond with them...I'm more connected to them, not that I don't care about the other patients-you must actually be dedicated for the ARV work, you can't go in there half-hearted."

Pharmacist, district hospital in urban setting

Social workers expressed that counseling grandmothers, who are frequently the primary caregivers of HIV-infected children, was one of the most challenging aspects of their work. For many grandmothers, knowledge of their grandchild's HIV status often occurs after a serious illness for which hospital attention is required.

The emotional and physical difficulties experienced by grandparents who are caregivers of HIV-positive children are serious concerns. Oftentimes while grandparents are still mourning the loss of their child, they must deal with the shock of an HIV-positive diagnosis of their ill grandchild, and then realize that the reason for their own child's demise was HIV infection. The social workers felt that they did not have the time to provide adequate psychosocial support (i.e., numerous consecutive individual counseling sessions) within the current constraints of the health service.

\section{Being undervalued}

Counselors expressed the perception that even though health care staff recognized their value as part of the care team, this attitude had not yet filtered down to patients. They suggested that the team be responsible for emphasizing the valuable role of the counselor within the care system.

"Patients are not educated about the value of the counselors. They think it's a waste of their time [being sent to us] - until they've had a session with us then they realize the value of having had the time with us."

Counselor, secondary care level in urban setting

“...it's a multi-disciplinary team effort that's going to [overcome challenge of being undervalued]. The doctors, the admin staff, the nurses, everyone needs to speak of the time spent with the counselor as if it's routine...not some optional thing on the side for freaks-it should be standard of care that when the patient comes in, they spend some time with the counselors to go over the big things, to monitor and check...."

Counselor, secondary care level in urban setting 
They also expressed feelings of frustration with regard to overcoming parental resistance to ARV treatment for their children.

“...some parents are very, very difficult. They don't believe in this ARV thing for their children, especially the fathers, they like to use the traditional thing. They don't believe in this thing because there are too many people who die because of this AIDS...."

Counselor, tertiary care level in urban setting

The extent of non-disclosure and denial, especially on the part of non-biological caregivers of HIV infected children, was further cited as a common challenge faced by social workers called upon to counsel patients.

\section{Physical and Structural Challenges}

\section{Lack of space}

A common concern among respondents was that current space and staff would become inadequate if the number of people accessing ARV services was to grow. The lack of clinic space for patient waiting areas and consulting rooms for clinical and counseling consultations was already a major problem for most facilities.

“Some clinics have enough space, some clinics don't—-this clinic doesn't have enough space, so they have to give me their [the nurses'] room on the day when one of us [doctors] are

consulting....”

Doctor, primary care level in rural setting

"...I have a problem of space in most of the clinics for counseling because there should be some private room and privacy which is not the case most of the time."

Doctor, primary care level in rural setting

“...space...sometimes people come [to assist with the care], you can have two clinicians and a dietician here [in this one room] with absolutely no privacy...."

Doctor, tertiary care level in peri-urban setting

Some health care workers indicated that the lack of space could be overcome in some instances if health authorities at institutions, especially at the tertiary level, supported the work of the pediatric HIV clinics.

" The challenge relates to getting internal support that it [is] a worthwhile intervention to do. We have no HIV clinic per se, so patients see us at different places on different days because we don't have a 'home,' the patients don't have a 'home.' We can't house all the services we can offer, such as [a family centered service] because we don't actually see parents and children in one physical space...so there isn't [coordination] of appointments either...."

Doctor, tertiary care level in urban setting

Pharmacists cited the additional problems of lack of adequate storage space for large quantities of pediatric medicines and no space for confidential counseling on ARV administration. 
"Pediatric ARVs are very bulky and require much more storage space in the pharmacy than adult ARVs."

Pharmacist, district hospital in rural setting

“...we can't actually take the patient into that room but I use that space to counsel them if there's a lot of patients in the queue behind them-I make sure they move away and you can see if a patient is looking around or anxious...but it's not that confidential...."

Pharmacist, district hospital in urban setting

However it was also pointed out that having separate counseling facilities available in the pharmacy for patients on ARVs could reinforce stigma.

"Other patients want to know why 'these' patients are going in."

Pharmacist, tertiary care level in urban setting

\section{Policy and Procedural Challenges}

\section{Drug policy, procurement, and supply}

There are fewer drug options for the treatment of children than of adults. In addition, procuring adequate drug supplies is problematic because it is difficult to predict the numbers of children requiring medication. As pharmacists and pharmacy staff noted:

“...I keep records...to help him see that now he has to order more because the numbers keep on going up on a daily basis. The minimum stock level won't be the same this month because the numbers are escalating."

Pharmacist assistant, tertiary care level in rural setting

“...at this stage we don't have a clue how many children come, some weeks you get 10 and the next none, so I don't know how to order the amount of stock."

Pharmacist, district hospital in urban setting

In addition, the fear of running out of drugs may lead to stockpiling, which can cause expired drugs to end up on the shelves. At the same time, because each child patient requires large amounts of antiretrovirals (mostly in syrup form), this can result in storage problems at pharmacies, as mentioned above, and limit the amount of drugs that can be ordered at a time at particular sites.

Existing weaknesses in health system management seriously affect the potential for the ARV rollout, as illustrated by the following quotation:

“...I mean they don't have even basic [stocks] — the stock levels of ARVs are just holding back the program completely. Many sites cannot start because they've no drugs while other sites can just maintain present patients on treatment. [We] haven't initiated a new patient on treatment since January because they don't get enough stock. There have been stock failures where existing patients on treatment didn't get medicine!”

Doctor, primary care level in rural setting 
ARVs are classified as Schedule 7 drugs, ${ }^{5}$ which some consider a barrier to rapid rollout because this requires the drugs to be ordered on a patient name basis. ARVs are rapidly becoming one of the common chronic drugs issued at health services, and respondents noted that this requirement is slowing down the pace of procurement.

“...you know there's no medicine needed by more patients at our facilities than ARVs...I mean Schedule 7 should be for items that few people will need, but this is the medicine most people need."

Doctor, primary care level in rural setting

\section{Record keeping}

There is no standardized data collection form for HIV management and treatment of pediatric patients. Each facility had its own system of record keeping and monitoring. Interviewers noted that most sites visited were using an adapted version of the MSF monitoring form developed for adult patients attending HIV clinic.

There was a sense among clinicians that there are child-specific elements that need to be captured and used for monitoring care and treatment among children, which are not factored into in the current data collection forms. One doctor expressed this:

“...we got forms for adults but for pediatrics we just have an 'initiation of treatment' form but thereafter we use the adult form and I think it will help if we have different forms for children. I think there are different things we need to ask them, the parents or caregivers, and you forget if it's not there or if there's not a space for you to fill it in. I think that is one thing that will help with the follow-up as well."

Doctor, primary care level in urban setting

\section{Socioeconomic Challenges}

\section{Poverty and welfare grants}

The Social Assistance Act 2004 (Republic of South Africa 2004b) provides the following types of grants for vulnerable children: a child support grant, a care dependency grant, and a foster child grant. Adults may receive a disability grant, an older person's grant, a war veteran's grant, and a grant-in-aid. As of April 2005 children 13 years and younger became eligible for a poverty-relief grant designed to help the caregivers meet the child's basic needs (Streak 2005). These grants range from R190p.m. ${ }^{6}$ (child support grant) to R820p.m. (old age pension and care dependency grant) (Manuel 2006).

\footnotetext{
${ }^{5}$ Act 90 of 1997, Section 22A stipulates, "a Schedule 7 substance shall not be acquired by any person other than the Director-General for the purpose of providing a medical practitioner therewith, on the prescribed conditions, for the treatment of a particular patient of that medical practitioner..."

${ }^{6}$ Conversion rate at time of study approximately $6.5 \mathrm{ZAR}=1 \mathrm{USD}$.
} 
People infected with HIV are disproportionately poor and marginalized members of society. This is a challenge to pediatric HIV care and adherence to treatment. Health care workers in general reported that they must ensure that caregivers can access social grants, which is often hampered by a lack of identity documents/birth certificates and the fact that children do not have legal guardians. Health care workers cited that they are often frustrated by the expectations patients have regarding social welfare grant applications, because patients often believe that their HIV-positive status automatically means that they are eligible for welfare.

“...parents feel their children automatically need to get a [disability] grant....”

Social worker, tertiary care level in metropole setting

“...no children [under twelve years] qualify for a disability grant-social welfare says they can't get till they're 12 years....”

Social worker, district hospital in urban setting

"I think social services need to be basically upgraded tremendously to support these children and their families who are on treatment, just like the issue of getting a mom an identity document so she can access a grant or basic things like that-that I can't help with but somebody has to help these people!”

Doctor, primary care level in peri-urban setting

The social worker is at the frontline of welfare-related work and found that the most overwhelming aspect of their work was related to the economic circumstances of the patients attending the health facilities. At the same time, one social worker expressed the opinion that the grants themselves are insufficient for HIV-infected patients, and that, in a sense, the grants were not considered worth the amount of effort that goes into the application.

"R170 is too little for them. They have to come to the clinics at least three times a month and they need special food."

Social worker, district hospital in urban setting

\section{Transport/distance to facilities}

The majority of children in the country are managed at secondary and tertiary centers or dedicated ARV centers for HIV treatment; these facilities are not necessarily located near where people live. This results in the cost of transport being a barrier to consistent attendance and even causing hardship, such as when people have to prioritize the expense of transport over other competing household expenses with a limited budget.

“...it’s expensive to maintain a regular appointment....”

Doctor, tertiary care level in urban setting

“...travel to the clinic [at the hospital] is a problem for the patients and they can't make it to the clinic, so therefore the clinic has to basically start going to them."

Doctor, primary care level in peri-urban setting 


\section{Changing caregivers}

Even if children have a biological parent or constant primary caregiver, a caregiver's sporadic work opportunities often result in several different people accompanying the child to the clinic for various appointments. This results in inconsistent messages to the caregiver as well as an inability on the part of the health care worker to obtain consistent patient history, which compromises the quality of care.

"...they are not always brought by their biological parents, there's always a change of caregivers, an aunt and the grandmother does not give the same information so every time we have to start from the beginning and have to explain what we have explained before...."

Doctor, primary care in urban setting

The ill health and/or death of parents can also impact negatively on a child's health care and adherence to medication. Multiple caregivers and instability of residence add to the challenges.

"A lot of our parents are very sick so that actually impairs their ability to look after the children."

Doctor, primary care level in peri-urban setting

"...it's very hard to check up on adherence ...you realize that the person who comes in with them [the children] is not the person who looks after them in terms of their medicines."

Doctor, primary care level in peri-urban setting

"...especially when the mothers die, [there are] multiple caregivers of the orphan children. Some of the children we don't know what happens to them, we just lose them."

Doctor, primary care level in urban setting

\section{Lack of community awareness and support}

Some sites reported that community members are not aware that an ARV service is available and that children can be treated. In addition, doctors perceived skepticism around ARV treatment of children in terms of the effects on the drugs on children.

"I don't think we've had enough education in terms of the access [to treatment]... and then we probably need to change the perception that ARVs are not good. I don't know how we can do that...it all started in the media, maybe we can go back to the media to change it...because ARVs to everyone is still a mystery-it's a mystery that people don't want to delve into...."

Doctor, tertiary care level in peri-urban setting

One clinician in a rural area raised an interesting theory regarding the community's prioritization of children for treatment:

"...the way I understand it is there's extremely limited resources and in a community that is very poor, that is kind of struggling to survive, sick children are a bit low on the priority list. Resources will be spent on the healthy child who is likely to survive and grow up to work for the family than the sick child who will likely die."

Doctor, primary care level in urban setting 
However, the marginalization of sick children does not only occur at the community level but at the health service level as well. Because more adults present for care, they consume the bulk of the staff time and resources as illustrated by the following quotation:

"...[We] cannot handle the load of what is expected of us for adults and children, the children have to wait-once in they're alright...."

Doctor, primary care level in urban setting

When asked what they saw as the major challenges to increasing access for children, most nurses stressed the need for community interventions such as public awareness campaigns and patient, caregiver, and teacher education regarding the availability and benefits of treatment for children. More than one respondent emphasized the need for information to give patients about the ARV program and how it can assist children in particular:

"I'm sure the ARV rollout started before community mobilization, especially in rural areas, because they don't know about ARVs, even if it's for the adult...most of these children you'll find that it's not their mothers who look after them, it's the grannies, it's the people who have no knowledge of HIV and AIDS...who didn't even know that the child is HIV-positive...."

Nurse, tertiary care level in peri-urban setting

"...Since the government decided to take steps in providing treatment we should be able to let people know and give them hope...."

Nurse, primary care level urban setting

“...through public talks-if you could get public talks, if we could get more, even the teachers at school, I think they can help us [create awareness ]...."

Nurse, primary care level in rural setting

\section{Lack of capacity to manage chronic and adolescent care}

Since ARV treatment is understood to be lifelong, children are expected to live long and productive lives. However, this also means the patients will require chronic health care intervention and will not leave the health care system. Rather, patients will constantly be added to the service.

"They [children] are complicated patients, it involves management of chronic disease-I mean these patients are going to be with us for a very long time. It doesn't look like the primary health care system and the district system would be able to look after these kids for a long period of time ...it would be ideal... a challenge to find a way of getting them to look after the children once they are stabilized."

$$
\text { Doctor, tertiary care level in peri-urban setting }
$$

The advent of ART and the shift to HIV being treated as a chronic disease poses challenges on a scale not previously dealt with by health services. Children now have a better prognosis of living with HIV and growing into teenagers and young adults. This calls for interactions between the health service provider, parent/caregiver, and child with regard to HIV and related matters on an ongoing basis and in an age- or developmentally-appropriate manner. 
Adolescents accessing services pose a challenge to health care providers. It is recognized that the approach toward them should emphasize their adherence to the lifelong therapy. The most common strategy adopted for providing services to adolescents was changing the adolescent-specific clinic times to the afternoons to allow them to attend school in the morning. Other activities included support groups, awareness programs, and youth camps held during school holidays in collaboration with communitybased organizations.

“...we're planning a support group for teenagers...we've just had so many challenges with them...the social workers are working on that."

Doctor, primary care level in urban setting

“...we've got a 'Love Life' [project] center where they receive awareness training in the form of videos etc...this is done in conjunction with TAC [Treatment Access Campaign].”

Doctor, primary care level in rural setting “...usually in winter holidays there's a school camp ...not for those on ARVs but a mixed group of infected and uninfected children...to build some kind of HIV awareness."

Doctor, primary care level in urban setting

HIV-infected adolescents often experience behavioral problems and intellectual and emotional delays, which require specific interventions. Some services with access to psychological and psychiatric services equipped staff to address these challenges.

“...we have our child psychiatrist come and meet with us once a month on a Thursday afternoon immediately after our school-going clinic to discuss psychiatric problems...but our main issue is behavioral problems rather than psychiatric. We've had the odd child with depression but I can count them on a couple of fingers..."

Doctor, primary care level in urban setting

Another challenge clinicians face is the need to address issues of sexuality and contraception with the children in their care, as expressed by the following respondent:

"Unavoidably we have to address the issue around sex education and family planning because there are some of them that we suspect are [sexually] active so we have to give them guidance...."

Doctor, primary care level in urban setting

Exactly how this education and guidance occurs within the consultation is not clear and there are reservations expressed that doing this in the presence of the accompanying adult could hinder open dialogue.

\section{Funding}

In the case of non-governmental sites or sites established primarily through donor/research funding (even within the public health sector), the obligation to ensure sustainable treatment to those already receiving ARVs constrained the rollout at these institutions, resulting in waiting lists and capping of numbers on treatment. 
“...because we are a purely funded site, we're very reliant on funding from external sources and that determines the number of patients that we can include in our [treatment] program."

Doctor, primary care level in peri-urban setting

In the public sector the issue of budget cuts was of concern to some managers in terms of jeopardizing the successful rollout of ARVs, as illustrated by the following quotation:

“... I think we're probably on track as to getting most things sorted out provided our funding doesn't get cut. That could happen tomorrow and then...let's say if they pull the plug tomorrow the whole thing [service] is just going to implode!"

Doctor, primary care level urban setting

\section{Examples of Overcoming Challenges to the ARV Rollout}

In addition to conducting interviews, researchers also recorded their observations of the study sites in detailed field notes taken during visits. The notes include descriptions of the facilities, waiting times, staff professionalism, and overall functioning of the sites. Although these narratives are impressionistic, they offer additional insight into the environment that health workers face during the ARV rollout, and provide details that help round out the information offered during interviews. The field notes highlight the many challenges and barriers that health providers must deal with, capture the satisfaction many get from their work, and detail creative ways they have found to sustain and even expand ART to children with limited resources.

\section{Sharing optimism}

Although during interviews some providers described their work as emotionally demanding, during fieldworker visits others expressed satisfaction, feelings of accomplishment, and hope.

"She [dietician] describes her work as enriching and that the sadness experienced when first seeing a child initiated on ARVs is quickly dispelled a few months later when it seems unbelievably to be the same child."

District hospital, Free State

"He’s [facility manager] an optimistic person and he confidently speaks about the successes achieved in Kayelitsha in terms of the ARV rollout."

Primary care clinic, Western Cape

\section{Overcoming staff challenges}

Despite staff shortages and other challenges faced by healthcare providers, some facilities found ways to support staff, such as by giving them opportunities to air their concerns and work through problems during regular meetings. 
"Weekly staff meetings with all staff members are conducted where issues concerned with running of clinic are dealt with. These meetings serve several functions including the provision of staff with information about the antiretroviral treatment program in the Western Cape; informing staff about national and international developments, and research developments and their implications for care, administrative, and logistical problems; and revisions to clinic protocols are discussed. In this way staff members are supported."

Tertiary hospital setting, Western Cape

Facilities offered varying degrees of training and mentorship to staff, as described in Chapter 4. Experienced doctors took the lead in offering training to other doctors and nurses.

"There is a hunger among the nurses for more training and education in HIV and ARVs."

Primary care site, Eastern Cape

\section{Overcoming patient/caregiver barriers}

Facilities often attempted to reduce the barriers to treatment for patients, such as the financial and physical burden of multiple appointments.

"This site has combined its adult and pediatric clinic, ensuring children and their caregivers synchronised appointments and avoiding additional financial and time burden to already impoverished families."

District hospital, Free State

In addition, some facilities were involved in income-generating projects, including vegetable gardens for added nutritional benefit to their clients and their families.

"The vegetable garden was pointed out to me...the vegetables grown are given to patients and the remainder gets sold to the community. Profits realized are used to buy new seeds and gardening necessities."

District hospital, Free State

"She [dietician] is in the process of formulating a proposal for a food garden. This would serve to teach patients skills about developing their own sustainable home food gardens and serve as a source of producing subsistence for home use."

Tertiary hospital, Gauteng

"There are small-scale income-generation projects, mainly beadwork. Beautiful items, ranging from jewelery to African garments, are made. Members are those who attend the support group meetings.”

Non-governmental district hospital, KwaZulu Natal

\section{Creating partnerships and links}

Facilities worked to stretch limited resources by forging partnerships and links with NGOs, universities, and other organizations. This pooling of resources strengthened treatment areas such as patient follow-up, 
counseling, data management, and respite care. Examples of these relationships were cited in notes from fieldworker observations:

"A home for HIV infected children, which is privately sponsored, is based on the hospital premises. This organization mainly houses orphans and children who have been removed from family to a place of safety by the Social Welfare Department."

District hospital, Free State

"Strong linkages exist between the ARV clinic and non-governmental organizations in the nearby vicinity. These are: an orphanage/day care center offering support both practically and emotionally for mothers and caregivers. They assist in finding placement for children after the death of their parents and provision of home-based care by trained caregivers. MSF has a support system going in Khayelitsha, and a whole array of support is offered. From training of counselors, to information provision and the training of support group members on HIV/AIDS including PMTCT...the demonstrated model of collaboration between government and NGOs, though not an easy relationship to manage, can be very successful at scaling up and ensuring sustainability."

Primary health care clinic, Western Cape

"A collaboration between the University of Cape Town and MSF is instrumental in establishing the data collection, monitoring, and evaluation system for HIV/ARV clinics established in this district. Until recently, when the provincial health department established data capture clerk posts for HIV/ARV data capture, MSF employed and supported the data capturers in all the HIV/ARV facilities. This database serves as a data entering point for both HIV and tuberculosis information. Data entering is done on a daily basis and patient attendance is also monitored in this way."

Primary health care clinic, Western Cape

"The five trained counselors are employed by Wola Nani, an NGO which entered into a collaborative partnership with the clinic. They act as interpreters and counselors to the pediatric and parallel adult service."

Tertiary hospital, Western Cape

Doctors and facility managers played a key role in creating these linkages, as described by one fieldworker:

"The director has also been instrumental in setting up a community liaison group, and according to staff, has always maintained involvement in the group and has been at the forefront of care for HIV-infected children. The director formed a relationship with a local children's home and served on their board of directors for four years while playing an instrumental role in advising this organization how to ensure access to treatment for orphaned children."

Tertiary hospital, Gauteng 


\section{Being innovative and forward thinking}

Many of the facilities visited were looking to the future and cited plans for adding space to the clinics, expanding services, hiring more staff, and taking other steps to expand the provision of ARVs to children. This includes research and programs intended to improve service provision in the long run.

"Data processing is also a major challenge, as just about no data being captured electronically, [the facility manager] feels that data capturing will minimize the workload. The latter can be done by having access to all hospital services from any terminal installed in a public service setting. The department is currently in the process of doing a pilot study where the facility visited will also be used as one of the pilot sites. The idea is that if information regarding a particular patient is captured, if he/she visits another hospital the tests already done do not have to be repeated. It is envisaged that this will save the department lots of money in the long term."

Tertiary hospital, Eastern Cape

"The facility manager is responsible for the coordination of the development of the Provincial Down Referral Guidelines for Children on Antiretroviral Therapy and is on the health information systems task team to support the process. He is the project manager for a multi-center collaboration, in partnership with a health care institution that intends to develop an evidence-based best practice model to improve the chronic care management of children with HIV in this area. He has also been playing an integral part in the implementation of a pediatric antiretroviral program at the tertiary hospital and a local clinic."

Tertiary hospital, Gauteng 


\section{Caregiver Survey}

Structured questionnaires were administered to 126 caregivers of children on ART. The interviewers collected information about sociodemographic characteristics, access to care, HIV infection in the family, diagnosis, referral and treatment, disclosure, and perceptions of services. A convenience sample of caregivers attending the clinic during the period of the fieldworker visit was interviewed, except in the Eastern Cape, where the fieldworker was accompanied to the home of the only child on ARVs in the village to interview the caregiver.

\section{Background I nformation}

The sample reflects the fact that the Western Cape and Gauteng provinces had the largest number of children on ART in this sample of facilities - approximately half of the respondents came from Harriet Shezi and Red Cross Children's Hospital. Both of these facilities served large peri-urban populations with high unemployment rates. Of the 126 caregivers that were interviewed, 77 percent of them came from households that had refrigerators, reflecting the urban or semi-urban location of the majority of them. The family sizes were relatively small with a median size of two adults and three children.

The vast majority of caregivers interviewed were female; two-thirds were mothers, 16 percent grandmothers, 8 percent aunts, and the remainder fathers, uncles, and volunteers. Ninety-eight percent of the respondents were the primary caregivers of the child they had brought to the clinic. Among the children in the care of the respondents, the median age was 4 years (range: 1 to 8 years) and 56 percent were boys.

When asked who provided financial support for the child, the largest proportion of caregivers mentioned the biological mother (51 percent), a grandmother (19 percent), and the father (11 percent). The remaining contributors (19 percent) were aunts, uncles, and foster parents.

The data show the important contribution of government grants to the financial support for the household. Of the 126 caregivers, 15 percent received no grant at all, 73 percent received one grant, and 12 percent received two grants. Fifty-nine percent of caregivers interviewed mentioned that they received childcare grants. Disability grants were received in 34 percent of households (in South Africa, disability grants are given to HIV-positive adults with CD4 cell counts of less than 400). Nearly a quarter of caregivers mentioned that they receive grants other than those mentioned above, likely child maintenance grants.

Children's adherence to ARVs may be impacted by both their living arrangements and their caregivers. During the week, just under a quarter (23 percent) of children whose caregivers were interviewed lived with both of their biological parents; 37 percent lived with their biological mothers, 15 percent with a grandmother, and 8 percent with an aunt. During weekdays, almost half ( 48 percent) of the children were taken to crèche for day care, and a significant number (14 percent) were taken care of by a grandmother. The rest lived with grandfathers, uncles, in a children's home, or with foster parents. Living arrangements were similar on the weekends. 
During the week, the key people responsible for administering medicines to children were biological mothers (52 percent), grandmothers (17 percent), either biological parent (15 percent), and aunts ( 9 percent). The same pattern was observed for those responsible for administering medicines at night and over the weekend.

\section{Referral}

Most of the children (71 percent) were referred to the HIV clinic from a community clinic, some of them without yet having had an HIV test. About a fifth (21 percent) were referred from the in-patient ward of a hospital, 6 percent from a private practitioner, and 2 percent from a PMTCT service.

The vast majority of children had been tested for HIV because they were chronically ill (44 percent) or hospitalized with a serious illness (40 percent), and another 6 percent because they were diagnosed with TB. A small minority were tested for reasons other than illness, namely, because the mother was participating in a PMTCT program (5 percent) and presumably was HIV-positive herself.

Figure 1 Reasons for child test $(n=126)$

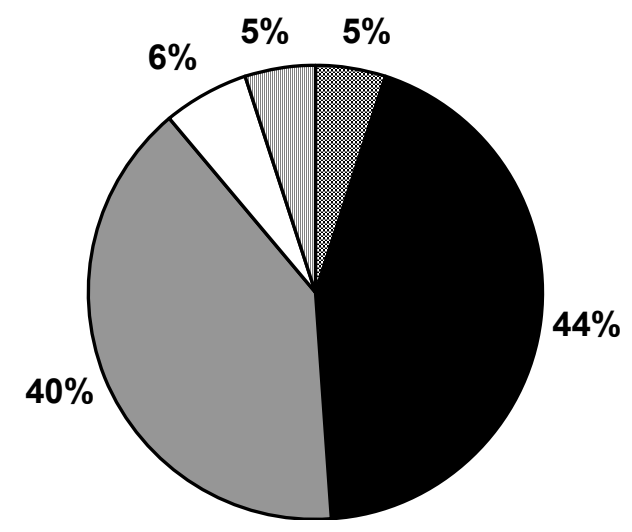

\begin{tabular}{|l|}
$\square$ PMTCT program \\
$\square$ Child chronically ill \\
$\square$ Hospitalization \\
$\square$ Case of TB \\
$\square$ Other
\end{tabular}

\section{Access to Treatment}

\section{Distance, time, and cost}

Access to ARV services was explored in terms of distance from and time taken to get to facilities offering relevant services, and the cost of services. On the day of their interviews, the vast majority ( 89 percent) of caregivers said they had used taxis to get to the health facilities. Only 7 percent walked, 5 percent used a bus, and 2 percent used a car. 
Figure 2 Distribution of time to source of ARVs $(n=126)$

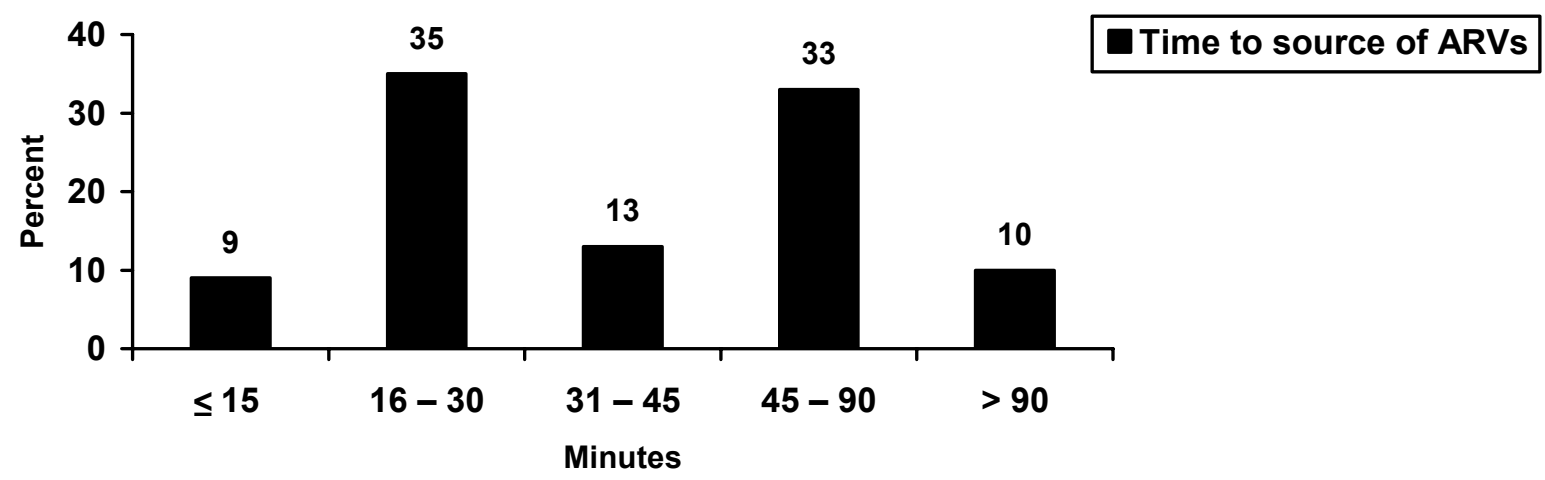

As shown in Figure 2, it took nearly 60 percent of caregivers less than 45 minutes to get to ARV services. On the other hand, 10 percent of caregivers had traveled two or more hours to get to the health facility. The caregivers had paid up to R100 for their transport with the median amount paid being R15. Although 17 percent of caregivers reported that they were charged fees for the child's treatment, further inquiry revealed that these charges were nearly all for the actual medication for children attending the nongovernmental health facility.

\section{HIV infection and concomitant health conditions}

Many families caring for HIV-positive children were already heavily burdened with HIV infection. Three out of four caregivers responded that there was at least one person in the household who was infected with HIV besides the child. Of this group, just over a half (52 percent) were said to be on ARVs. Though 56 percent of those on treatment received ARVs from the same clinic as the child, only 38 percent of the group had the same appointment dates as the child. The majority ( 86 percent) of caregivers preferred household members to have their appointment dates on the same day.

Children who were taking ARVs were reported to have other health problems. For example, their caregivers reported that 26 percent had TB, 4 percent had asthma, 2 percent had epilepsy, and 10 percent had other ailments. In addition to ARVs, many children were taking cotrimoxazole ( 76 percent), multivitamins ( 70 percent), and TB drugs (18 percent). Most caregivers (92 percent) mentioned that they received other medications for the children in addition to ARVs at the ARV pharmacy.

\section{Adherence}

A common problem associated with ARVs is lack of adherence to dosing schedules. Nearly all (96 percent) of the children had reportedly been given ARVs on the morning of their clinic visit. According to the 126 caregivers, 15 percent of the children had missed medications during the previous week; reasons included the child's poor health status, the caregiver had forgotten, the time had passed, the child had vomited, or the medications were finished. Caregivers used different mechanisms as reminders to administer medications on time, including cellular phone alarm systems (35 percent), TV programs (10 
percent), and watches/clocks ( 3 percent). Other caregivers considered administering the medication part of their daily routine ( 46 percent) or were reminded by the child ( 6 percent).

\section{Disclosure}

In 88 percent of cases, family members (most often the mother and grandmother) in the household knew about the child's HIV status. Teachers and neighbors were generally not taken into confidence about the HIV status of the child. Reasons for choosing not to disclose included fear of stigmatization and a desire to keep the status of the child secret from others.

Figure 3 People who know the status of the child $(n=126)$

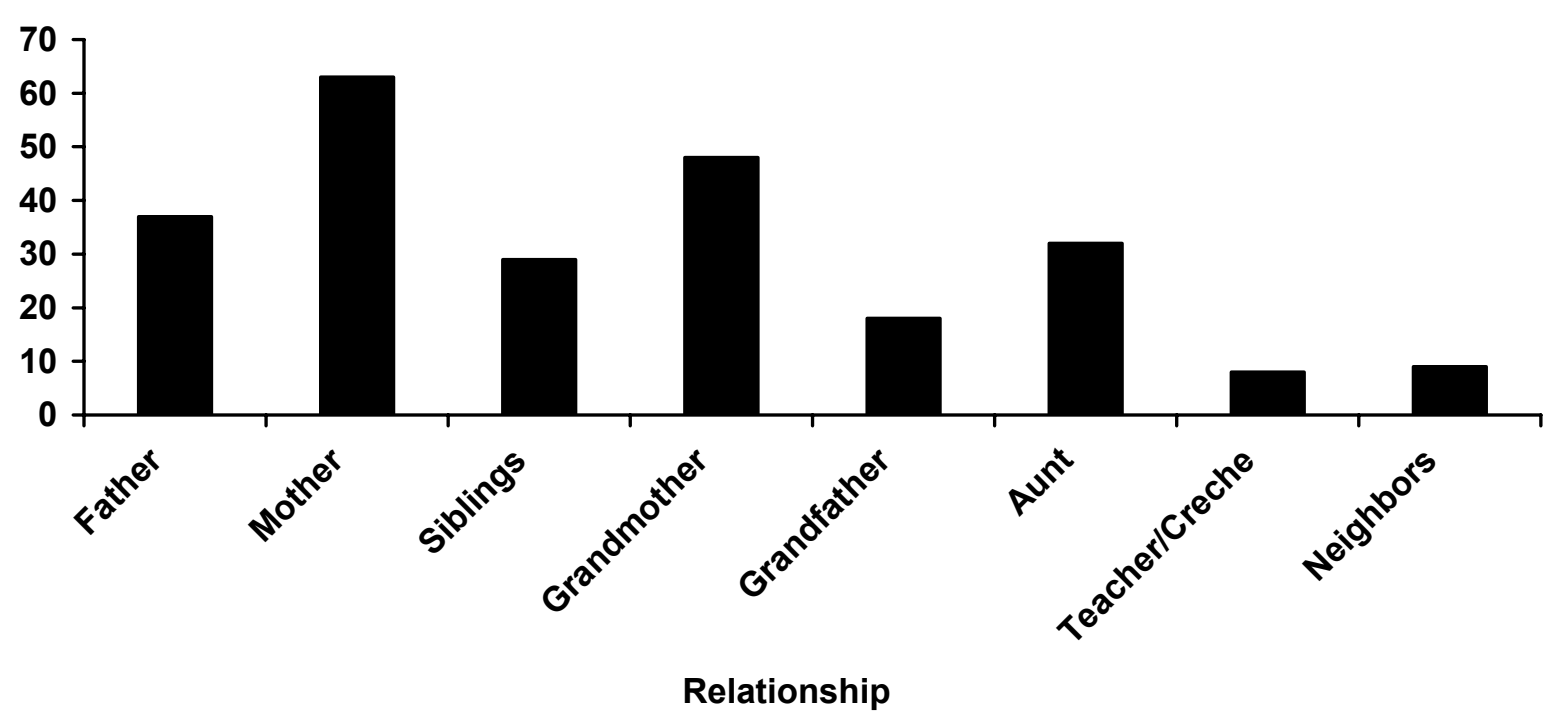

Only 21 of 126 caregivers reported that the children in their care knew their own HIV status; the ages of the children were given as five to eight years (two were reported as two and three years old, possibly an error in reporting). Caregivers generally believed that the child should be informed of his or her HIV status between the ages of 6 and 10 years.

\section{Perception of Quality of Services}

Caregivers were generally satisfied with most quality of care aspects. For example, all respondents indicated that the provider addressed their questions and concerns satisfactorily and spent enough time with them. The respondents, however, felt that they could have been provided with more information (Table 8). Caregivers' interaction with pharmacy staff also scored positively. Respondents indicated that the medicines were explained to them very clearly and that they were given tools, primarily syringes and medicine cups, for administering them. 
Although the quality of services was ranked high both during interactions with providers and pharmacists, it is important to consider the general courtesy bias associated with responses to questions on quality of care in the context of African cultures.

Table 8 Caregiver responses to questions on information needs $(n=126)$ Caregiver communication needs Percentage

Caregiver had questions or concerns about ARV or other issue 71

Would like to receive more information in form of pamphlet 97

Would like to receive more information in form of video 58

Would like to receive more information in form of discussion groups

78

Would like to receive more information in form of talks in the waiting room

84 


\section{Conclusion and Recommendations}

The South African government approved the provision of ART in the public sector as part of a comprehensive strategy to combat HIV and AIDS in November 2003. Since then there has been a strong focus on extending ART access to patients in the public sector. At the end of August 2005, more than 50,000 South Africans were receiving ART within the public sector.

The pediatric ARV rollout is lagging behind the adult ARV rollout in South Africa, as fewer resources are allocated specifically to the pediatric program and child-specific elements are not prioritized at the policymaking level. In many settings in South Africa children are still not included in ARV programs. A policy review revealed that current guidelines for the rollout pay insufficient attention to children.

There are currently few research studies describing the services for infected children in South Africa. To highlight the areas of the pediatric ARV rollout in South Africa, a rapid situational analysis was conducted in 16 study sites across five provinces. These sites, which varied from primary to tertiary level clinics, reported a total of approximately 1,300 children on ARV treatment by the end of March 2005, with approximately 60 percent under the age of six years. A total of about 180 interviews with health care workers and caregivers of children were conducted, with the majority of health care workers comprised of medical doctors and nurses. In addition, the facility managers of all sites were interviewed and detailed field notes were recorded.

The study highlights the variation of models of care for HIV-infected children in South Africa, given the differing geographical and structural challenges as well as management and human resource capacity in the various provinces. For example, the composition of staff at the sites varied in size, with health teams ranging from 3 to 20 members. What has emerged from this study is the conclusion that there is no "one size fits all" model of pediatric care and treatment service. However, enthusiastic and dedicated individuals have demonstrated that the human resource factor is the most critical component of any model of care. The dedicated doctor who forges links and partnerships with other health care workers and organizations to successfully start a service, sometimes with little or no backing, is an example of how an individual is capable of making a difference. However, the role that MSF and other donors have played in the establishment of treatment centers for children should not be overlooked. MSF has demonstrated that they can replicate their peri-urban model of care (Khayelitsha) in a deeply rural area such as Lusikisiki, with equal success.

Across the study sites, the health care workers who were interviewed shared their perceptions of the shortcomings and successes of the pediatric ARV rollout, shared common concerns, and cited similar challenges. Many of the barriers to the rollout emerging from this study relate to the current constraints of the health services infrastructure, such as lack of staffing and space (i.e., for waiting rooms, consultation, counseling, and pharmaceutical storage).

The government drives the ARV rollout program in South Africa. It is therefore imperative that certain elements are put in place by the government to ensure that children are placed on the ARV rollout agenda. This includes ensuring a specific pediatric budget for allocation of child- specific resources within the general health service, such as staffing, pediatric skills training for all health care workers, and pediatricspecific equipment. 
The following recommendations emerged out of the interviews and stakeholders meetings.

\section{Build an Effective, Comprehensive PMTCT Program}

There is consensus among health care workers that preventing pediatric HIV infection through a comprehensive PMTCT program remains the most effective strategy to modify the course of the pediatric HIV epidemic in South Africa. There is limited integration between PMTCT and ARV programs for children in the country. In this study, PMTCT programs were not frequently cited as a source of referrals of children for HIV care. Furthermore, strengthening the links between pediatric and obstetric services is important for optimal functioning of PMTCT programs.

Based on the current South African birth cohort of approximately 1 million, between 83,000 and 90,000 pediatric infections of the current total of 104,963 could have been be prevented. This would reduce the pressure on the public health service and consequently have a significant positive impact on the quality of existing clinical services for HIV-infected children.

\section{I dentify HIV- infected Children}

The lack of waiting lists for the treatment of infected children can be attributed to a failure of the systems that identify infected infants and children in a timely manner. HIV DNA PCR diagnosis for infants starting at age six weeks is not being accessed in many parts of the country. Currently, 85 percent of infants with HIV are lost to follow-up from the PMTCT program and an estimated 40 percent of these infants die during the first year of life without treatment. Diagnostic strategies for children require urgent attention.

Many HIV-exposed or -infected children are not receiving cotrimoxazole prophylaxis, nutritional support, or other important interventions. Children are started on antiretroviral therapy in the advanced stages of HIV-infection, often after they are hospitalized with severe medical complications. Early identification and management may prevent many of these admissions. Community-based programs, especially those offering routine immunization and nutrition support, can play a key role in early diagnosis.

\section{Train Health Professionals}

In this study, the most commonly cited challenge was the lack of trained staff, in particular, nurses skilled at diagnosing and caring for pediatric infectious diseases. Doctors and nurses repeatedly mentioned the need for additional pediatric-specific training, which is essential to overcome staff resistance to working with children and to lessen the reliance on scarce physicians with pediatric specializations. Many nurses reported an eagerness to learn new skills and some specifically requested additional on-the-job training to help balance the workload and expand services.

It is therefore recommended that as a direct extension of its treatment guidelines, the HIV/AIDS Directorate in the Department of Health develop uniform training programs and manuals for all health cadres. A review and revision of the content of training materials for student nurses and medical students 
should be undertaken and curricula should be consistent with the Department's treatment guidelines. All externally funded treatment programs should base their treatment programs and their staff training on the Department of Health's training guidelines. The African Network for the Care of Children Affected by HIV/AIDS developed a training program based on its recently published handbook. Some countries, including Tanzania, Kenya, and Uganda, have incorporated aspects of this program into their national training programs on pediatric HIV infection. A similar process is required in South Africa to improve the quality and consistency of care that HIV-exposed and -infected children receive.

\section{Monitor Services for HIV-infected Children}

Accurately collected statistics are essential for understanding the extent to which the country has responded to the pediatric HIV epidemic and will be important for guiding the future allocation of resources and training. This is particularly pertinent with respect to the pediatric ARV rollout, as many ARV sites do not yet provide access to children in need of treatment. At present the total number of children on ART in South Africa is not known but estimates range from 5,000 to 7,500. Similarly, the total number of children who currently require antiretroviral therapy is not known.

It is therefore recommended that statistics be collected routinely from all sites in South Africa, to identify areas that are either not treating children or are falling below acceptable enrollment rates. These areas can then be targeted for appropriate support and training.

\section{Utilize Models of Care}

The development of models of care for children should take into consideration local conditions and constraints. These may range from child-centered, adolescent, and youth-friendly services to familycentered care. A family-centered model is ideal and feasible at a primary care level. Health districts should be encouraged to develop "bottom up" solutions based on local circumstances. Models of care should include the integration of services for HIV-exposed and -infected children and adolescents including PMTCT, ART, STI, and TB services.

\section{Promote Collaboration Between Clinical Services and Non-clinical Governmental Sectors, Community-based Services, and Health Systems}

Pediatric HIV infection is a complex disease affecting families in a variety of ways. Comprehensive management requires optimizing health service delivery as well as promoting collaboration between clinical services and several other governmental sectors, community groups, and NGOs, particularly service providers, around related issues.

The Department of Health should play a lead role in bringing together groups and guiding the development of management areas that require inputs from other sectors. Key issues that should be addressed through collaboration include: (1) social support and interventions for children without regular or reliable caregivers willing to be responsible for medication administration and clinic attendance; (2) educational support for the special educational needs of HIV-infected children with learning disabilities; 
(3) collaboration between clinical services and community-based services such as home-based caregivers (to assist in identification of at-risk children not in care; (4) communication and information dissemination to increase levels of HIV treatment literacy in communities, especially child- and adolescent-specific treatment literacy; (5) inter-governmental and government-to-civil society collaborations with respect to relevant legislation, policies, and planning; and (5) human resource assessment (particularly the scope of practice of nurses in pediatric care) and planning.

\section{Meet the Special Needs of HI V-infected I nfants}

Treating infants (i.e. children less than one year of age) with ART is a particularly challenging task. Many pediatric ART programs have primarily enrolled children who are over two years of age. This study revealed that many children younger than six years were being treated at tertiary facilities due to the lack of expertise at primary level facilities. Most of these children accessed care and treatment at the tertiary facility due to an acute illness but were not being referred out for chronic care. It is assumed that many infants who need ART are in fact being missed or dying before their HIV infection is identified.

Many factors contribute to the complexity of pediatric HIV management. For example, infected infants often have additional medical problems, knowledge of the use of antiretroviral treatment in the very young is incomplete, drug dose calculations in the very young may require the use of body surface area calculations, suspensions have to be administered to young children, and obtaining blood samples for monitoring young children is technically challenging. For these reasons consideration should be given to capacitating institutions at all health care levels with expertise and clinical competence to manage young children. Specific planning for the treatment of infected infants will help to reduce the high mortality in children less than two years of age, a distinct feature of the epidemic in Africa.

Community-based ARV programs need to diversify the functions of these programs to include clinical monitoring (clinical and CD4 count monitoring), cotrimoxazole and INH prophylaxis, nutritional support, anti-TB treatment, routine immunization, treatment of minor illnesses, and appropriate referral and endof-life care.

\section{Encourage Adherence}

In order to achieve the goal of ART - survival and improved quality of life - near perfect adherence is required. This study raised the challenge of adhering to dosing schedules. Caregivers reported that 15 percent of the children had missed medication in the previous week. The study found that there were no consistent methods of adherence monitoring used at sites or between sites. Pharmacists were mainly relied upon to provide this monitoring function (which at some sites included defaulter tracking).

It is recommended that a national pediatric adherence strategy guideline be developed that includes treatment literacy training specifically targeted to pediatric patients and their caregivers, as well as the provision of resources for medication administration tools. 


\section{Adolescence: Support the Transition from Childhood to Adulthood}

An emerging feature of pediatric ARV treatment is adolescents who require a specific strategy to overcome the challenges of adherence. As access to care and treatment for infants and children improves, the number of teenagers who acquired HIV through vertical transmission will increase in Africa.

Currently, health care workers are reporting that issues such as disclosure of the child's status, coping with their reaction, and treatment adherence are major challenges.

Current monthly statistics do not give an indication of the extent of ART treatment in adolescents. This should be changed so that the provision of services for youth can be monitored and improved. The special needs of adolescents should be accommodated at all institutions treating children.

Measures to prevent infection in adolescents should be improved through consistent and age-appropriate education, post-exposure prophylaxis services, reducing rates of teenage pregnancies, prevention and treatment of sexually transmitted infections, and awareness and availability of contraceptive devices.

Ensuring greater access to treatment for children depends on placing children prominently on the ARV rollout agenda and identifying those at risk at early stages, which includes an effective PMTCT service and referral link with child health services. It also requires that all health care workers are vigilant so that HIV care and treatment is not the domain of only a specific group of health care workers. 


\section{References}

Centre for Actuarial Research and South African Medical Research Council. 2004. "The demographic impact of HIV/AIDS in South Africa-National indicators for 2004." http://www.mrc.ac.za (accessed 30 March 2005).

Dorrington, R.E., et al. 2004. The Demographic Impact of HIV/AIDS in South Africa. National indicators for 2004. Cape Town: Centre for Actuarial Research, South African Medical Research Council, and Actuarial Society of South Africa.

Eley, B., et al. 2004. "Initial experience of a public sector antiretroviral treatment program for HIV infected children and their infected parents," South African Medical Journal 94:643.

Giese, S., et al. 2003. CI and National Department of Health. Helping children in a time of HIV and AIDS. http:// www.sarpn.org.za/documents/d0001579/HelpingChildren.pdf

Health Systems Trust. 2005. South African Health Review.

http://www.hst.org.za/generic/item.php?item_id=29

Horizons and University of Cape Town Faculty of Health Sciences. 2005. "Exploring current practices in paediatric antiretroviral roll-out and integration with early childhood programmes in South Africa: A rapid situational analysis," Horizons Research Update. Washington, DC: Population Council.

Luo, Chewe. 2005."Access to ARVs, the big picture," presentation at the Pediatric AIDS Treatment for Africa Conference, Cape Town, South Africa, 29 November. http://www.one2onekids.org/

Manuel, Trevor A. 2006. Minister of Finance, SA Budget speech.

http://www.fin24.co.za/BudgetSpeech2006.pdf

Newell, M-L, et. al. 2004. "Mortality of infected and uninfected infants born to HIV-infected mothers in Africa: a pooled analysis," Lancet 364(9441):1236-1243.

Office of the Global AIDS Coordinator. 2004. "The President's Emergency Plan for AIDS Relief: U.S. Five-Year Global HIV/AIDS Strategy.” Washington, DC: Office of the Global AIDS Coordinator.

Provincial Health Department of the Western Cape. 2005. "ARV statistics for March 2005," personal communication with Dr. Peter Bock in May.

Shungking, M. and M. Zampoli. 2004. What about the children? A discussion paper on the antiretroviral rollout in SA. Children's Institute, UCT.

http://web.uct.ac.za/depts/ci/pubs/pdf/rights/facts/Save the Children ARV FINAL.pdf

South Africa Department of Health. 2004. "Strategic priorities for the national health system (20042009)." South Africa: Department of Health. http://www.doh.gov.za/docs/policy/stratpriorities.pdf (Accessed 10 October 2005). 
South Africa Department of Health. 2003. "Operational plan for comprehensive HIV and AIDS care, management and treatment for South Africa." South Africa: Department of Health.

http://www.gov.za/docs (Retrieved 5 July 2005).

South Africa Department of Health. 2002. "Policy framework for non-communicable chronic conditions in children." South Africa: Department of Health.

South Africa Department of Health. 2001. "Policy guidelines for youth and adolescent health." South Africa: Department of Health. http://www.doh.gov.za/docs/policy/yah/index.html (Accessed 20 October 2005).

South African Vitamin A Consultative Group. 1994. "Anthropometric, Vitamin A, iron and immunization status in children aged 6-71 months in South Africa," South African Medical Journal 86(4):354-7.

Streak, Judith Streak. 2005. "Government's social development response to children made vulnerable by HIV/AIDS: Identifying gaps in policy and budgeting." Pretoria, South Africa: Institute for Democracy in South Africa.

Tshabalala-Msimang, M. Statement of cabinet on a plan for comprehensive treatment and care for HIV and AIDS in South Africa, 2003. http://www.info.gov.za (Accessed 14 November 2004).

UNAIDS. 2005. AIDS Epidemic Update, December 2005. Geneva: UNAIDS. URL:

http://www.unaids.org/epi/2005/doc/report_pdf.asp (Accessed 18 April 2006)

van Kooten, Niekerk, et al. 2006. "The first 5 years of the family clinic for HIV at Tygerberg Hospital:

Family demographics, survival of children and early impact of antiretroviral therapy," Journal of Tropical Pediatrics 52(1):3-11.

Watson, D. C. and J. Farley. 1999. "Efficacy of and adherence to highly active antiretroviral therapy in children infected with human immunodeficiency virus type 1," Pediatric Infectious Disease Journal 18(8):682-689.

WHO. 2003. "Treating 3 million by 2005: Making it happen.” Geneva: World Health Organization. 


\section{Appendix}

\section{Site Visits-Fieldworker Observations}

Below are the narratives of observations and impressions written by the researchers visiting the study sites. The research team consisted of two doctors, one professional nurse, and two HIV-trained counselors. Fieldworkers were asked to make observations and to document their experiences during the 2-3 days they spent at a site to conduct interviews with health workers and caregivers. Since the sites varied greatly, they were not given formal guidelines for note taking, but asked to focus on how well each site provided ARV services to children. The notes include descriptions of the clinics and pharmacies, staff, and overall functioning of the sites. Although these narratives are impressionistic, they offer additional insight into the environment health workers work in during the ARV rollout, and provide details that help round out the information offered during interviews. Any statistics presented have not been verified.

\section{Tertiary Hospital Site Visit-Harriet Shezi, Gauteng Province}

\section{General overview}

In response to the overwhelming HIV epidemic and the burden that it was placing on hospital services, a pediatric HIV out-patient service was established in 1997 by one of the doctors at this tertiary hospital, and later this clinic was selected as an international pediatric research site in collaboration with a perinatal research unit (hereafter referred to as "the Group").

The hospital clinic site was established in 1999 to standardize management of HIV-infected children seeking care at the three academic hospitals in the greater city hospitals. There are three primary level care clinics situated in the large peri-urban catchment area of the hospital, which form part of the academic training circuit. The Group has been responsible for treating more than 1,000 children with ARVs since the start of the government rollout program in April 2004. Despite the geographical separation of the units, the Group has successfully demonstrated a strong collaboration between the different sites. Considering the population density and the HIV prevalence rates in this province, and especially the immediate area in which the Group operates, the unit has one of the largest cohorts of HIVinfected infants, children, and adolescents in the country.

Gauteng Province had an HIV prevalence rate of 33 percent among antenatal clinic attendees in 2004, which is the second highest HIV prevalence rate in the country. There are approximately 10,000 patients on ARVs in the province. The Harriet Shezi clinic served approximately 550 patients on ARVs in March 2005 , while approximately 650 HIV infected patients were receiving follow-up at the clinic. Followup/repeat scripts caseloads are increasing, resulting in waiting lists for new patients. An estimated 130 children are targeted for ARV initiation by April 2006.

\section{Infrastructure and setting}

The waiting room of the pediatric clinic is surrounded by colorful pictures and near a lovely garden with tables and chairs where some caregivers just relax or simply play with their children. There is a notice 
displayed on the wall reminding caregivers of their next support group meeting. On the day of our visit, a television is on and some children are playing in the play corner.

The clinic is comprised of one pediatrician, three medical officers, two IMCI trained nurses, one primary health care trained nurse, one staff nurse, six counselors, a data manager assisted by two data capturers, and a unit manager. In addition, there are two doctors, the director/facility manager, who see patients when the clinic is very busy. On the days that I was present, the facility manager had a number of patients scheduled.

The clinic sees at least 40-80 patients a day. Formally booked patients are seen three days a week and on other days any patient who turns up without an appointment is still seen by any doctor available at that time. There are plans to develop a facility where adults and children with HIV infection and/or TB coinfection can receive care at a single center. An adolescent-friendly antiretroviral service for HIV-infected youth has also been established at this clinic.

\section{Patient route of entry/access}

Most patients are referred from the pediatric wards of the tertiary hospital. The Group feels that by doing this, no patient who qualifies to start ARVs is missed in the hospital system. Although scaling up the ARV treatment program and outreach community care centers is one of the major aims of this clinic, they feel that they are still not reaching all children in need of treatment.

\section{Community networks and involvement}

The director has been instrumental in setting up a community liaison group, and according to staff, has always maintained involvement on the ground and has been at the forefront of care for HIV-infected children. The director formed a relationship with a local children's home and served on their Board of Directors for four years while playing an instrumental role in advising it on how to ensure access to treatment for orphaned children.

A support group has been established at the clinic and is facilitated by an outreach counselor. The counselors are responsible for follow up of patients who default. A major problem voiced by the counselors is lack of transport in order to effectively conduct home visits. On a daily basis, different support group meetings are being held including one-on-one counseling, caregiver education, and income generation groups. During a discussion with various staff members regarding the income generation groups, however, most feel they are not working as they had envisaged.

\section{Staffing}

The facility manager is responsible for coordinating the development of the Provincial Down Referral Guidelines for Children on Antiretroviral Therapy and is on the health information systems task team to support the process. He is the project manager for a multi-center collaboration, in partnership with a health care institution that intends to develop an evidence-based best practice model to improve the 
chronic care management of children with HIV in this area. He has also been playing an integral part in the implementation of pediatric antiretroviral programs at the tertiary hospital and a local clinic. He is actively involved in patient care, despite his many other managerial responsibilities.

A recently qualified pediatrician joined the clinic at the beginning of the year and has a keen interest in working with HIV-infected adolescents. Another doctor who joined the team a year ago is involved in staging and treatment clinics that assess and prepare children to begin ART.

An additional doctor had resigned and was leaving at the end of the month. There was no doctor to replace her yet (according to the facility manager). She has been instrumental in training staff from the clinic in pediatric ARV work. She also performs clinical duties at the site clinic on ARV clinic days.

There are two IMCI trained nurses as well as other nurses, all who seem very efficient and professional with their patients and caregivers.

The social worker has a room for private and confidential counseling of caregivers. She is employed on a full-time basis and her work includes follow up of the non-adherent patients and providing ongoing counseling. She forms part of the community liaison group, which is flourishing and active at the clinic.

\section{Nutritional support}

A dietician runs this clinic three days a week with the assistance of another dietician from the tertiary hospital. Unfortunately they have to share space with a doctor or counselor, which results in patients and caregivers not having any confidential interaction with health care workers. She is in the process of formulating a proposal for a food garden to teach patients skills for developing their own sustainable home food gardens. Her main challenges are related to the poor socioeconomic status of the patients, who often require a longer supply of milk from the PMTCT scheme, particularly for children under one year of age. She has a large ARV patient load and in addition sees other children in the general pediatric wards.

\section{Blood taking and lab services}

A sister and a nurse who assist in the weighing room take blood, but children under age two are referred to the laboratory's phlebotomist. I observed that the doctor takes the bloods of children who are participating in the research study. The tertiary hospital's porters collect all bloods at different times throughout the day and processing is done at the hospital lab. Virology and CD4 results are readily available from the computer at the clinic, which is linked to the lab for accessing blood results.

\section{Pharmacy services}

A pharmacist runs the clinic pharmacy in addition to assisting in the main pharmacy of the institution. The clinic pharmacy is very small and ARVs are dispensed from there. It is very private and has a lockable gate, which only the pharmacist can access. There is a small room where she does ARV drug counseling before issuing ARVs. 
Patients participating in the research get ARVs at the clinic pharmacy and all other medication from the main pharmacy. Non-research patients get both their ARVs and other medication from the main pharmacy, which extends the clinic visit period for patients quite a bit. The main pharmacy is just outside the clinic and has a clerk who takes patients' scripts to the pharmacy assistant and issues the patient with a follow-up date. The pharmacy assistant prepares the medication in the pill picking area. Then, either the clinic pharmacist or the main pharmacist give patients the prepared meds and counsels them. Counseling takes 20-30 minutes per patient. If any further problems are experienced the patient is referred to the counselor who assists the pharmacists. This counselor is specifically trained by the ARV rollout team from the provincial health department to deal with these issues. All patient scripts are copied, filed, and captured, by the clerk.

The pharmacist at the main pharmacy is responsible for procuring all pharmacy stocks, including ARVs. The clinic pharmacist and the main pharmacist are the only people who have keys to the storeroom and dispensing pharmacy. All ARVs are supplied from the local store's medical supply depot and ordered online on a monthly basis. The clinic pharmacist then orders from the main pharmacy store on a weekly basis.

\section{Local community clinic}

Accompanied by one of the clinic doctors and counselors, we took the 25-minute drive to the community clinic, which lies at least $16 \mathrm{~km}$ from the tertiary hospital. The community clinic doctor is about to leave and is not being replaced, supposedly because the staff there has not shown any enthusiasm for acquiring ARV pediatric skills. (It seems though, from my observations, that it would be almost impossible for them to do anything other than attending to their own adult clinic, which is full to capacity.)

At this community clinic, there is only one medical officer, who as the day progressed had not had time for any kind of break; after I interviewed her at about 13h30, she continued working. According to her she would like to be involved in the on-site pediatric ARV training, but there just is no time.

Currently there are only six children on ARVs who are being followed up by the clinic doctor and counselor (from the tertiary hospital - if they should terminate their services at the clinic, those patients will be referred back to it). Children who are accompanying parents simply do not get diagnosed and are left untreated. Time did not allow for me to interview any other staff members, which included two nursing sisters who are primary health care trained, but seeing adult patients on ARVs only; one nursing sister who is in charge of the blood taking; and one counselor assisted by the counselor borrowed from the clinic on Wednesdays. A driver transports all bloods from this community clinic to the tertiary hospital lab once a day.

\section{Main challenges}

While in my opinion an excellent service is being delivered at this site, all involved complain of the lack of space. I have seen how counselors and the dietician have to consult from the same room at the same time. Patients and caregivers seem nervous and distracted, which could compromise comprehension and possibly impact on ARV adherence. 
The pharmacists are experiencing staffing shortages but it seems that with the current constraints, it will not be possible to redeploy any more pharmacists to the site from the tertiary hospital staff. In addition, the constraint of submitting to Government-determined tenders for ARVs has resulted in shared tenders, leading to different medication packaging (colors and containers change), further confusing patients. They feel that this is particularly exacerbated when the person who collects the medication is not the person who administers it to the child.

\section{District Hospital Site Visit-Bloemfontein, Free State Province}

\section{Background}

The province has a population of approximately 2.7 million and a HIV prevalence rate of 30 percent (2632 CI 95 percent) among antenatal clinic attendees (2004). The ARV program started in May 2004, with approximately 1,000 patients on ARVs by March 2005, of which approximately 100 were children.

\section{General overview}

This site is a secondary health care level hospital based in a community district with many friendly people offering a comprehensive service to HIV-infected children and adults. The combined adult and pediatric clinic ensures children and their caregivers receive synchronized appointments, preventing additional financial and time burden to already impoverished families.

The staff is comprised of an ARV facility manager, a pediatrician, five medical officers (three experienced in ARV/HIV care and two inexperienced), two registered nurses, a social worker, a dietician, a pharmacist (another one on standby), and two ward clerks. The clinic has a spacious, though sparsely furnished, waiting room and large counseling and consulting rooms.

\section{Patient route of access to ARV services}

Patients are referred to this ARV clinic from the pediatric ward, three local assessment sites (one of which is on the grounds, but not affiliated to this ARV site), a secondary hospital (which has the role of start up site, rather than treatment site), and a children's home.

\section{Description of ARV clinic staff}

The facility manager has many other duties including surgery, which were assigned to him prior to filling this post as ARV manager.

There is a senior specialist pediatrician employed by a tertiary hospital directly affiliated to a University. She spearheads the pediatric ARV program not only for this district but also for a number of other districts. Her role encompasses training and supporting staff, running the pediatric ARV clinic at the site and its three assessment sites. In addition, she is currently also involved in setting up ARV satellite clinics 
for other community clinics. Every Monday she spends a full day at this district hospital site performing clinical duties with most of the children booked for the ARV clinic. The medical doctors perform the same duties under her guidance - consequently, most children with complicated opportunistic infections and ARV drug-related complications are booked for a Monday.

There is an apparent insecurity among the medical officers about their ARV management skills and consequently they rely on the support and mentorship of the visiting pediatrician.

A very short and informal interview with one of the nursing managers revealed the anxiety among nursing staff regarding phlebotomy on young children. She defended her nursing staff, saying that it is not in any nurse's scope of practice to take bloods from a child's jugular vein, and that her nursing staff in the pediatric ward has also expressed their concerns regarding their lack of ARV rollout training. They have feelings of insecurity and immense inadequacy and even though "The Centre for Excellence" has a 24hour hotline to support staff, she felt that without basic knowledge in this field, a hotline is not much help. She feels that training in pediatric HIV management skills is a priority.

A common theme among doctors was that they felt their present experience with regard to ARV/HIV management was not sufficient to handle certain complications among children. They were astonished to hear of the hotline and were unaware of this service.

There was only one professional nurse on duty during my visit, since the chief professional nurse was on accumulative leave and the other professional nurse had been temporarily seconded to a home-based caregiver program at a local clinic. She approached each caregiver and child with friendliness and her interaction can well be described as patient-centered. She expressed fears of ineffective patient management should there be a marked increase of pediatric patient enrolment into the ARV program, due to a shortage of nursing staff. It seemed though, that she was not very involved in clinical duties, but rather spent most of her time fulfilling clerical duties, and because of her non-involvement with phlebotomy, she felt no need for further training. Her understanding is that the medical and nursing health authorities were in mutual agreement that nurses should not take any bloods from children younger than six years old.

The social worker works for the entire institution as well as the ARV unit and the majority of her time is spent securing disability grants for the children-liaising directly with the Department of Social Services. She estimated that she could see up to 10 patients a day.

An informal interview was conducted with the dietician. She collaborates extensively with clinics, NGOs, and developmental services in the surrounding vicinity. The children's caregivers are given a supplement for their diets, breastfeeding is largely encouraged, and vegetable food packets are given at each visit. She describes her work as enriching and says that the sadness experienced when first seeing a child when it is initiated on ARVs is quickly dispelled a few months later when she sees the improved condition of the child.

The district has home-based caregivers directly affiliated with the three assessment sites who see them as a great help.

The pharmacy is situated on the ground floor with a bench outside it for patients who are waiting for general medications and ARVs. There are three iron barred windows through which patients have to 
speak to pharmacy staff; to the side is a corner where the pharmacist speaks to ARV patients about their drugs with absolutely no privacy. The ARV pharmacy has a slam lock gate and door. There is no phone in the pharmacy, but there is an air conditioner and a normal sized fridge with a temperature log hanging on the door. Only the three ARV-trained pharmacists have access to this pharmacy. The key is locked up in the chief pharmacist's office. There are numerous shelves for drugs and files. The main pharmacist at the institution is in charge of the ARV rollout program in the district. During her absence she is replaced by two other ARV rollout-trained pharmacists. The drug order is calculated from the amount of new patients seen the month before with the addition of the amount of patients already on ARVs. The facility is in the process of building a bigger pharmacy directly attached to the ARV clinic, to be completed by end of this year.

A privately sponsored home for HIV-infected children is based on the hospital's premises. This organization mainly houses orphans and children who have been removed from families to a place of safety by the Social Welfare Department. Children are referred to this home from the ARV clinic, the pediatric ward, and occasionally the three assessment sites. Two registered nurses and a staff nurse take care of all ARV-related duties along with four assistants, a cook, and a cleaner. They have bed capacity for 25 children and during our visit there were 18 children. Only two of the 18 children are on ARVs, although the staff feels more children actually need them. Reasons cited for not having children on treatment include the waiting procedure and the fact that some do not meet criteria for ARVs yet (some of which have to do with social support rather than clinical status). The doctors, who also work as pediatricians in the pediatric ward in the district hospital, make sure children get additional medical treatment from the hospital.

\section{Assessment sites}

It was early morning when the pediatrician drove us through the peri-urban area, heading toward a township about $11 \mathrm{kms}$ from the district hospital. Our first clinic stop was a large clinic, which operates as a primary health care center, with radiography, pharmacy, well baby center, acute and curative care, and the ARV clinic. The curative and acute care department has its own doctors and IMCI trained nurses and the bulk of the referrals to ARV clinic happen from there. They showed me the vegetable garden, and it looks healthy and well maintained. The vegetables grown are given to patients and the remainder gets sold to the community. Profits realized are used to buy new seeds and gardening necessities. The other two clinics are just $5 \mathrm{kms}$ apart from each other and are much smaller in terms of service delivery.

\section{Major challenges expressed by health workers}

The slow progress of the pediatric rollout in the district was blamed on the focus on adults rather than children and the lack of pediatric-specific resources. Nurses need training and confidence in phlebotomy skills (especially for children under six years of age) and in that way could assist in decreasing the already overburdened workload of doctors in the ARV treatment clinic.

Currently nurses are not taking bloods on all children younger than six, which impacts negatively on the screening process for ARV readiness. The children have to be screened by doctors in the ARV clinic, sent back to the fully booked drug readiness program, and then back to the ARV clinic to initiate their ARV 
treatment. Home-based caregivers are not coping with the required home visit follow-up function, resulting is many children being lost in the system. This is compounded by frequent changes in caregivers and the expensive trips to and from the various clinics.

Staff shortage is cited as an endless problem, as all current staff working in the ARV clinic at this site are still expected to fulfill clinical duties in other areas of the institution. Of particular concern is that when a doctor is out sick in another department, a doctor from the HIV/ARV clinic is borrowed, irrespective of the workload in the HIV clinic. This could cause the perception that HIV/ARV care for both adults and children may not enjoy similar preference in relation to other hospital services.

\section{Final comments}

To sum up, this district has a small percentage of children on ARVs in comparison to adults. The systems and physical resources are in place, but trained human resources remain a problem. The other major stumbling block was the inability, or reluctance, of registered nurses to perform phlebotomy procedures on children younger than six. It seems that the nurses' skills in the current pediatric ARV clinics are underutilized in terms of ARV management. In my opinion, administration and completion of documentation seems to take precedence over all other clinical care duties.

\section{Primary Care Site Visit-Lusikisiki, Eastern Cape Province}

\section{Background}

The estimated district population is 150,000 . The 2004 antenatal HIV prevalence was 23 percent. With an estimated overall prevalence of 10 percent, there are probably at least 15,000 HIV-positive individuals in the district. However, this number does not include those who access care from out of district. The roads into the town are all tarred now (although seriously potholed), so it is not surprising that HIV positive people from surrounding small towns travel to this town to access HIV care.

\section{Structure and organization of district health service}

This district is served by 12 comprehensive clinics, three district hospitals, and a provincial hospital. Of the 12 clinics, two are in town, one of which is essentially part of the provincial hospital's outpatient department that was relocated to a primary center in the past two years. The clinics are nurse-driven and offer comprehensive services: both promotive and preventive (immunizations, under-5 clinic, nutrition, family planning, and antenatal) as well as curative (TB, sexually transmitted infections, minor ailments, and chronic conditions).

For the past two years, the clinics have offered an HIV service, which includes PMTCT, VCT, provision of Pneumocystis Carinii Pneumonia (PCP) prophylaxis, treatment of opportunistic infections, and more recently, antiretroviral treatment. 
The clinic staff run the HIV service with support from the mobile team of MSF doctors who visit each clinic at least once a week. The doctors initiate antiretroviral treatment and see "problem" follow-ups, while the nurses and counselors manage the rest.

The antiretroviral component of the HIV program was started by the NGO at the provincial hospital at the end of 2003. In the next year, the NGO will hand over the hospital HIV unit to the hospital staff and will focus their efforts on provision of antiretroviral treatment from the clinics in a phased fashion.

At the time of the visit all 12 clinics have adults on ARVs, while eight have children on ARVs; other than the busy clinic attached to the provincial hospital with 16 children on ARVs, the rest have one to three pediatric clients at this stage. Together with 12 pediatric clients at the hospital, the children on ARVs total 39. There are a total of 99 HIV-positive children seen regularly at the clinics. There is no waiting list - the other children do not yet qualify for treatment.

\section{Staffing}

The hospital HIV unit is staffed by six counselors, a data capturer, an enrolled nurse, an enrolled nurse assistant, a matron (professional nurse), a pharmacist, and a doctor (who also works in the wards). The hospital social worker is supported by designated HIV funding. Both the social worker and pharmacist have only been appointed this year. There are eight other doctors, including a pediatrician, at the hospital - most are foreign.

At the clinics, the district employs a total of 39 nurses of various ranks and 60 counselors. As far as could be ascertained, few of the nurses have clinical training. They are expected to be generalists, able to perform any of the required functions. There are five counselors at each clinic, two of whom are responsible for VCT. The issue of their payment is controversial: the counselors are regarded as "volunteers" and are paid an allowance every six months, as the monthly amount falls below the minimum wage. The NGO supports the doctor, and has one clinical nurse in the field. While we were there, they were interviewing for a second such nurse. There is currently no district pharmacist to cover the clinics. There is one pharmacy assistant, and 11 others in training, five of whom are employed by the NGO. The only social worker for the district does not fall under the Health Department.

\section{HIV Day Unit-Provincial Hospital}

We spent the first morning at the provincial hospital and met with HIV Day Unit staff and our NGO guide. The Unit is a small wooden structure on the grounds of the hospital. It has three rooms - an office, a room with four beds, an entrance room with three beds, and a waiting bench outside. There are also two adjacent corrugated structures - the first has a waiting room and an office that the doctor uses to consult. Patients are referred to the unit from the outpatient department or come if they have an ARV-related appointment. The nurses see clients, put up IV lines, consult the doctor to order tests, etc. There is oxygen (face mask), an automatic BP machine, and a pulse oximeter. The pharmacist or an assistant dispenses ARVs and other medication at the unit.

We observed activities at the HIV Day Unit and interviewed a doctor, an enrolled nurse, the pharmacist, the social worker, and a counselor who is also a caregiver for a child on ARVs. 


\section{Clinic R}

This is the busiest clinic, with the largest number of clients on ARVs, including 12 children. On one side there is the main clinic with patients waiting outside under cover; on the other side is the newer NGOdonated ARV container. It has two consulting rooms, one of which is used by counselors if there is not a second doctor. The small waiting area inside this one fills up quickly, and the clients queue outside.

It is a busy day and most of the HIV clients are seen in the main container by the nurses and are only referred to the doctors once they qualify for ARVs. Later on in the day, we see a support group in progress on the lawn. A big circle of people (men and women, young and old, well and visibly ill) are sitting, standing, listening, and talking.

\section{Special event}

It was a public holiday and MSF had organized a party for the children on ARVs and their caregivers. Client education was really impressive. There is a big emphasis on knowing the names of the ARVs. Even the children can tell you what they are taking, as was evident at the "party testimonies." The local Treatment Action Committee (TAC) branch, the NGO, and the support groups have created songs to help them learn these things: they have a TB song (listing parts of the body that can be affected), a RIFAFOUR song, an STI song, and so on. One key observation was that there were no young men at the party; however, one dignified grandfather was in attendance with his little granddaughter, having lost both his daughter and son-in-law to AIDS.

\section{Final comments}

There is a problem of "finding" children who need ARVs because there is no waiting list. It became clear that PMTCT is not really working; areas of concern include if access to the dry-blood-spot PCR will increase the identification of positive children before they become symptomatic. Notably, nurses did not identify PMTCT follow-up or children on TB treatment as potential sources of pediatric ARV candidates. They instead expressed the need for more community outreach and mobilization, but they feel too overloaded to take that on.

There is a desire among the nurses for more training and education on HIV and ARVs. However, the facility manager feels that the nurses lack the basic knowledge and skills to perform their other functions, and this impacts on their ability to learn what is required to function in HIV and ARV management. One can't help wondering what the plans are, if any, to increase the nursing levels at the clinics given their heavy workload.

The overall provincial functioning is still very problematic, especially with respect to drug supply (particularly pediatric drugs and formula milk). The first day we were there, the hospital pharmacist had to drive for hours to the city to the medical depot to fetch ARVs. There is also the issue of the nonaccreditation of the pediatric component of the clinic sites. 
The facility manager has the notion that sick children are not seen as a priority in resource-constrained families, rather the investment is in adults or well children. The community sees no need to prioritize children, even at the clinics.

\section{Primary Health Care Clinic-Nolungile Clinic, Khayelitsha, Western Cape Province}

\section{General overview}

The study researchers visited one of three sites providing ARV treatment to children. This facility was chosen because it had the most experience in pediatric ARV treatment and the largest number of children on ARV treatment since the start of the program in 2000.

The grounds are very big and consist of a day hospital and well baby clinic. Today it is Wednesday, which is both adult and pediatric ARV clinic day. The rest of the week is used mainly to see the adult HIV-positive patients, although children are not sent away if they need to be seen. There is a pediatrician dedicated to the ARV clinic on Wednesdays and problem cases are mainly booked for these days. The ARV services at the clinic are jointly run by MSF and the Provincial Administration of the Western Cape (PAWC). The doctor who coordinates the ARV service at the facility is employed by MSF. The ARV clinic has two full-time doctors, two part-time doctors, one experienced HIV/ARV pediatrician, two professional nurses (not IMCI trained), and four counselors. They do not have a dedicated pharmacist or social worker assigned to the ARV clinic. The social worker that is employed by PAWC does, however, lend support and the staff may refer patients to her whenever she is available (bi-weekly, mornings only). Due the absence of a dedicated pharmacist, the doctors and professional nurses dispense all ARVs to adult patients. The doctors see all the children and therefore, the dedicated doctors do all the dispensing of pediatric ARVs.

Health education is an ongoing process, with a flurry of interactive activity taking place in the waiting area just outside the clinic rooms. Patients are listening and intermittently asking questions that are answered by the counselor. The doctors and nurses see up to 40 patients a day. Adult and pediatric folders are mixed together in the pile, and patients are seen on a first come, first served basis. Moms and babies are seen together if they are both here on the same day.

According to one of the managers, nurses are the true forerunners of HIV care in Khayelitsha (for adults) and it is sad that government authorities view them as the weakest link in health care. He feels strongly that recognition should be given to nurses both professionally and in remuneration. He speaks proudly of all the health care workers at various sites in Khayelitsha and it is evident that they are well on their way to even greater successes. The new adult HIV and TB clinic is pointed out to me and it looks good. He also paid great tribute to a pediatrician from the tertiary hospital that has been supporting their pediatric program since its inception, for training all his doctors in pediatric HIV care and for her ongoing mentorship.

The facility manager works at this facility full time, and sees both adult and pediatric patients. The commitment that this doctor and other MSF doctors display is immense. He's an optimistic person and he confidently speaks about the successes achieved in Khayelitsha in terms of the ARV rollout. He feels that 
it would help the workload of the doctors a great deal if there were pediatric HIV/ARV trained nurses to help with the HIV/ARV clinic. Although this is seen as a priority area, he says that neither PAWC nor Local Authority has plans to implement the services of IMCI-skilled nurses in the ARV program.

The trained HIV/ARV (hospital-based) pediatrician who provides expert pediatric consultation on a monthly outreach basis at each of the clinics has been working with the staff in these facilities for a number of years. The province has restructured the ARV outreach program from academic hospitals and she will soon be replaced by another doctor. She also feels that the site will do much better with the assistance of ARV pediatric trained nurses.

The role of the counselors at the site is mainly one of ARV adherence monitoring support and delivering health education on an ongoing basis. Every Wednesday a support group is held, where caregivers are able to express their fears and opinions about HIV and treatment management. Counselors have received training from both TAC leaders and MSF training coordinators. They also offer home-based care follow up of patients who are or threaten to be lost to the system.

\section{Referral points}

Strong linkages exist between the ARV clinic, community health centers, tertiary hospitals, and nongovernmental organizations in the nearby vicinity. There is an orphanage/day care center offering both practical and emotional support for mothers and caregivers. It provides and assists in placing children after the death of their parents.

MSF has a support system going in Khayelitsha, and a whole array of support is offered. MSF works in direct collaboration with TAC, with activities ranging from training of counselors, to information provision and the training of support group members on HIV, including PMTCT.

In general, the support that is provided by the community outreach programs situated in and around this area is phenomenal and definitely recommended for other sites.

\section{Data collection and capturing}

A collaboration between the University of Cape Town and MSF is instrumental in establishing the data collection, monitoring, and evaluation system for HIV/ARV clinics established in this district. Until recently (when the provincial health department established clerk posts for HIV/ARV data capture), MSF employed and supported the data capturers in all the HIV/ARV facilities). This database serves as a data entering point for both HIV and TB information. Data is entered daily and patient attendance is also monitored in this way. The professional nurse orders new ARV drugs on a weekly basis (60 percent from MSF and 40 percent from PAWC at the time of the visit). 


\section{Conclusion}

Because this is one of the sites at the forefront of PMTCT in the country, according to the consultative workshop report (January 2005), children are theoretically not lost in the system. Although most of the doctors working at the site believe that the figures for children enrolled in ARV treatment are still low in comparison to figures for adults, the conclusion reached is that children must have been lost due to not being tested in PMTCT (MSF implemented the early PCR testing prior to the government making it available in this province to overcome this problem). It is further surmised that the rapid progressors have died and are therefore not seen at the clinics in the district.

I feel strongly that the passion and dedication of the MSF staff was instrumental in inspiring the progress in this province with regard to HIV/ARV management. They have had a formal relationship with the provincial health department and have jointly worked on a plan to sustain the services and scale up in the district. The demonstrated model of collaboration between government and NGOs, though not an easy relationship to manage, can be very successful at scaling up and ensuring sustainability.

\section{Tertiary Hospital Setting-Red Cross Hospital I DC Clinic, Western Cape Province}

\section{Level of care}

Tertiary care facility performing primary and secondary functions.

\section{Model}

A comprehensive clinical service model for children, including access to the complete range of subspecialist services and funded from the provincial HIV/AIDS budget. This is complemented by a parallel clinical service for infected parents and caregivers funded by Absolute Return for Kids (ARK), an NGO.

I made several trips to the hospital to see various health care workers on different days. The outpatient building of the hospital in which the infectious diseases clinic (IDC) operates is a relatively new, multipurpose facility built with privately raised funds. It is apparent that it is a children's hospital, with outside gardens and play equipment, inside play areas, bright, eye-catching wall colors, window draping, and beautifully mounted pictures on the walls.

The infectious diseases outpatient clinics, which all include a large antiretroviral component, run three times per week. These comprise two general clinics and one clinic exclusively for school-attending children and adolescents. The outpatient service is augmented by an inpatient service, which consists of ad hoc consultation service throughout the week and a dedicated inpatient meeting on Monday afternoons. At this meeting new inpatients are considered for ART, and patients who are on ART with medical or surgical problems requiring admission and antiretroviral naïve HIV-infected children with complex medical conditions are discussed and their treatment reviewed. This forum is also used as an opportunity to educate ward consultants, registrars, and senior house officers about all aspects of the management of pediatric HIV infection. HIV/ARV care is specialist doctor-driven, with one clinical nurse 
practitioner, five counselors, hospital support staff, and access to the complete range of pediatric and surgical sub-specialist services including an onsite dental service.

It appears that a lack of adequate and dedicated space is a major problem for this clinic. In planning the new outpatient building about 10-12 years ago no consideration was given to infectious diseases or HIV infection. Consequently, the infectious diseases outpatient clinic service has no specific site within the new outpatient building but instead utilizes other specialist clinic space on clinic days. As one of the health care workers remarked, "We and the patients have no home." However, there are plans to build an additional wing to accommodate the infectious diseases outpatient activities. Counseling space is shared with the adult ARV service run by ARK and the Clinical Research Unit situated close to where clinics are conducted. Support group sessions for caregivers and activities for infected children are accommodated in other areas of the outpatient building close to where clinics are conducted. The numbers of patients seen per clinic are limited by waiting times at the pharmacy and pharmacy hours.

ARV and other HIV research/clinical trials are run in the Clinical Research Unit. Much of the HIV/ARV clinic staff is involved in research.

On-site training and mentorship of medical students, doctors, and other hospital, community, and visiting health professionals in HIV infection and antiretroviral therapy occur during clinics. Pediatric community outreach programs are run by some of the clinic staff members. At the end of March 2005 staff members were supporting pediatric ARV clinics in Langa and Gugulethu, two high prevalence health districts in the Cape Metropolitan Region, and were engaged in discussion about supporting several other community clinics.

\section{Staffing}

Levels of medical staff are high and most of the medical staff is pediatric specialist trained. There are five specialist pediatric and infectious disease doctors with pediatric registrars and students passing through for training. There is one clinical nurse practitioner with specialist training in ARV and HIV management. However, nursing staff members who support the clinics are drawn from the general, outpatient nursing pool. They are not permanent, but a few of the more regular nurses have acquired a fair amount of knowledge about HIV infection and antiretroviral therapy. A research nursing sister is currently providing a phlebotomy service on clinic days.

The five trained counselors are employed by Wola Nani, an NGO that entered into a collaborative partnership with the clinic. They act as interpreters and counselors to the pediatric and parallel adult service. Two community service dieticians provide a service on a referral basis but are not dedicated to the clinic. The social work department is available to the IDC but there is no dedicated social worker for the IDC. There is currently no clerk dedicated specifically to the IDC. The service makes use of the general outpatient clerical staff. Research staff members are called upon to fill staffing gaps when necessary.

Children who attend the IDC may access other hospital services on a referral basis, such as physiotherapy, developmental clinic, the dental service, dermatology clinic, and a wide variety of other specialist clinics. The clinic supports many researchers focusing on HIV and related issues, such as occupational therapists, physiotherapists, social scientists, post graduate students, and clinical researchers. 


\section{Pharmacy services}

The ARV dispensing function is incorporated into the main pharmacy which has been given two additional pharmacists posts for dispensing ARVs. The main pharmacy was recently renovated and expanded. It now has a dedicated child-friendly counseling room for the ARV pharmacists to assist with counseling and education for children starting antiretroviral therapy and to provide ongoing counseling to caregivers.

ARVs and other medication for caregivers/parents are privately funded by ARK and dispensed by ARKfunded pharmacists from the research pharmacy located in the Clinical Research Unit. This pharmacy is a registered pharmacy, and is generally used for dispensing research/clinical trial medication.

\section{Strengths}

I have noticed many strengths at this site, which could serve as lessons to other similar sites.

- The site has the ability to provide both tertiary and primary level care at one service point.

- The staff and IDC management are directly involved in the planning and execution of community outreach and the establishment of primary care ARV services in district/community health services.

- It is consciously gearing up for future exclusive tertiary care roles within the health system.

- It has high staff levels and resources, and a high level of ARV clinical care expertise.

- The flow of the clinic is well organized, although congestion does occur and space is limited.

- Adherence counseling and support is a central focus of all staff members.

- It includes holistic HIV and ARV care resources of other hospital services including occupational therapy, physiotherapy, and neurodevelopment care.

- Weekly staff meetings with all staff members are conducted where issues related to running the clinic are dealt with. These meetings serve several functions, including providing staff with information about the ARV treatment program in the Western Cape; informing staff about national and international developments, research developments, and their implications for care; addressing administrative and logistical problems, and discussing revisions to clinic protocols.

- It facilitates relevant research and utilizes the information for improvement of service and management.

- It serves as a training facility for local and visiting clinical health professionals.

- It provides a parallel clinic for HIV-infected caregivers.

- It offers structured support programs: counseling for individuals/group counseling for parents and caregivers.

- There is access to a social worker for advanced counseling needs and help with grant applications.

- There is access to funds for transport and food parcels through the hospital's charity organization.

- Good networking systems with outside NGOs and organizations to facilitate patient follow-up and respite care. 
However, the following challenges remain:

- Distance from patient and caregivers residences to the hospital.

- Limited intensive pre-initiation of ARV counseling (plans to overcome this are underway).

- Delays at the main pharmacy.

- No designated space for the clinic (plans to overcome this challenge are underway).

- Clerks and nursing staff are not permanently assigned specifically to the IDC clinic, resulting in periodic changes (staff recruitment underway to overcome this).

- Space constraints compromise counseling activities.

- Not all counselors are specifically trained in ARV treatment counseling.

- The main pharmacy is physically distant from the clinic and a source of stigma because only patients who are issued ARVs are called into the separate counseling room. These activities are, however, done discretely. Bottlenecks at the pharmacy slow down the processing of patients and are not ideal circumstances for patient drug counseling and adherence checks. (The planned outpatient upgrade will include a dedicated pharmacy.)

There is general consensus among the staff that the general care and monitoring (clinical staging and CD4 percentage/count) for HIV-infected children and ARV treatment programs for children should be expanded and strengthened at the primary/community level so that appropriate care, including ARV therapy, can be initiated before children develop advanced disease or life- threatening complications which require hospitalization. In addition, the referral links between PMTCT programs and primary health care clinics should be strengthened, to ensure that HIV-infected children have greater and appropriate access to services at community/primary health care facilities.

My overall impression was that this facility is a pleasant, child-friendly environment and that the staff has expertise to share with others that would increase children's access to ARV therapy.

\section{Tertiary Level Hospital-East London, Eastern Cape Province}

\section{Introduction and general overview}

This is a tertiary level hospital and is situated in a district. The HIV/ARV clinic is situated in the general pediatric outpatient department. The pediatric HIV/ARV clinic is commonly known as the "consistency clinic." The walls of the clinic are covered with tattered posters and information on HIV, patient rights charter, and Batho Pele principles. Most of the posters are either handwritten or simply photocopied, in an effort by staff to inform and educate patients. Pediatric ARV services are rendered on Wednesdays and Thursday afternoons between $14 \mathrm{~h} 00$ to $16 \mathrm{~h} 00$. One medical doctor (senior specialist pediatrician) and two professional nurses are running the clinic (one nurse was away on sick leave at the time of our visit). There are a number of student nurses at the general pediatric outpatient department, but they do not assist with the ARV clinic apart from weighing all children coming to the clinic.

In contrast, the adult ARV clinic is situated in a separate section quite a distance from the pediatric section. They have pleasant facilities such as a reception area, waiting room, and counseling roomshowever, these are partitioned rooms with no ceilings and therefore limited privacy. This section has 
more staff allocated, such as counselors, nurses, clerks, etc. There are plans for improved facilities within the hospital and the pediatric clinic will be moved adjacent to the adult ARV clinic in future.

There is only one full-time doctor initiating pediatric ARV treatment at this facility. Other doctors in his department are being mentored and provide backup service in his absence. The organizational policy regarding decisions on staffing and resources are reportedly highly centralized and staff here feel that their requests regarding the provision of extra doctors, nurses, counselors, a pharmacist, and a social worker are not being prioritized by those in authority. The service is completely government funded and the turnover of pediatricians is also seen as a problem because this facility offers training, after which doctors leave the service. The perception is that no one wants to work in an HIV-only pediatric setup. Downward referral of pediatric patients to the primary care level is virtually non-existent in this town, because the pediatric ARV rollout training for primary health care nurses has not started. The PMTCT service is not in full operation and therefore the prompt referral of HIV-positive children in need of ARVs is not happening. According to staff at the hospital, quite a large number of HIV infected children in need of ARVs have already died in the nearby vicinities.

\section{Patient follow up and referral}

The majority of patients are referred from the pediatric wards and by the time they reach the ARV clinic for appointment, they have been staged according to WHO criteria and the necessary immunological test results are available. There are referrals from other secondary and tertiary hospitals outside the city, and while no child is refused treatment, this creates follow up problems for patients in these outlying areas. When these children are referred back to their communities there are no follow up services or ARV accredited facilities, so they have to find their way back to the facility. Although this is working at the moment, its long-term prospects as envisaged by staff are bleak. General practitioners in town refer patients occasionally, as do the community clinics. When patients are not started on ARVs, they are referred back to their respective places with a letter stating their reason for not having started (such as psychosocial or failing the drug readiness program).

\section{Monitoring, adherence, and counseling}

According to the staff, adherence is excellent. This is based on the premise that all bottles are empty upon return for follow up and becauset they have only had two deaths of children on ARV treatment. All adherence problem cases are referred to a skilled counselor (nursing sister) who is in charge of the HIV/ARV counseling service, and are followed up on a biweekly basis or until all adherence problems are resolved. Routine caregiver counseling, however, is not done by a dedicated ARV counselor but rather by the nurse working at the pediatric HIV/ARV clinic, who also acts as an interpreter and assists where necessary with drug readiness counseling of parents/caregivers. She also assists with adherence monitoring whereby containers are checked, pills are counted, and the importance of taking ARV medication regularly is reiterated. All parents/caregivers are asked to demonstrate how much medication they draw up with a syringe and then they have to explain how they do it and at what times.

Although the ARV clinic is rather small in terms of patient follow up, they have about 70 children on the ARV program, with an equal proportion of male and females. The youngest child on ARVs started at age two months and the oldest child is 12 years old. Not many patients under six months of age are referred. 
Support given to children is minimal in comparison with the services ongoing for the adult patients. They see between 12-14 booked patients a day and occasionally an un-booked patient will present. The data collected is not stored on a database; any notes go into patient folders and the doctor keeps a separate file from which he can compile statistics for reporting purposes.

There is a pharmacist assigned to the pediatric ARV dispensary but there is no social worker or nutritionist. Social work or nutrition services can only be accessed with effort and referral and thus are not readily available to the pediatric service.

\section{Laboratory services}

Bloods are taken by the doctors only. The porters collect these bloods throughout clinic days, but not later than $4 \mathrm{pm}$. The laboratory inside the hospital will run all viral loads and send them to the laboratory virology department in the administrative capital; CD4s are sent to another city where they are flow cytometrised using the Beckman Coulter method for processing. Viral load results take at least one week to be available and CD4 counts take 3-4 days.

\section{Pharmacy services}

The ARVs were not locked away, but were out on shelves and every other staff member (in addition to ARV pharmacy staff) was freely accessing the pharmacy. This practice was explained by the fact that there is just not enough space dedicated to the storage of ARVs and that the store is overflowing. There is, however, a small lockable cabinet where they are locking some ARVs away. There is also an electronically controlled fridge that keeps the temperature between $2-8 \circ \mathrm{C}$. The pharmacist procures the three-month stock on hand from the main depot located in another city.

Challenges highlighted by pharmacists at this facility:

- There is no procurement policy in place at the facility.

- Short dated stock, e.g. Ritonovir, will be delivered and two months later it will be expired.

- Decanting pediatric suspensions into $500 \mathrm{mls}$ bottles "coke type plastic bottles" is a problem. These bottles are intended to save the patient/caregiver from having to leave with numerous $200 \mathrm{ml}$ bottles, but they are quite unwieldy, especially for families where there is more than one child. These families have to travel with public transport, which could cause the bottles to break.

- It can take up to 30 minutes to prepare medication for one patient, and there are not enough hours in one day for a single pharmacist to fill all scripts. Decanting the pediatric formulations also takes time.

The following observations were made at the pharmacy and confirmed by the pharmacy assistant:

- A register of all pediatric patients started on treatment is kept and recorded in MS Word on the computer at the pharmacy. The following fields are captured: patient name, date of birth, gender, folder number, ARV start date, drug regimen, and "additional information," under which column the last visit date and the next clinic dates are recorded.

- A solution of Stavudine powder is made at the pharmacy using distilled water and decanted into $500 \mathrm{ml}$ brown bottles for those patients who have fridges. The exact amount required $+20 \mathrm{ml}$ extra 
is given as a standard issue on all syrups. For those patients who don't have fridges, Stavudine capsules are issued with instructions to open these and dissolve into $10 \mathrm{mls}$ of tap water.

- All meds are decanted, even the Ritonivir and Kaletra. The reason given for the decanting practice was that "patients can't open the bottle caps."

- All brown bottles are labeled with different colored labels by type of medication.

- According to the pharmacy assistant, patients do not wait long at the pharmacy. When they go, they hand in their folder with the script, then the doses are checked and new labels with dosing instructions are written up. Each patient is counseled privately in a counseling room in the pharmacy that is specially dedicated to ARV counseling/teaching.

Interviews with health administrators not based at the facility revealed that they believe that ARV services should not be segregated from the rest of the services provided at the hospital. According to them, the stumbling blocks are the scarce resources, e.g. staff (all categories), equipment, and infrastructure. Data processing is also a major challenge, as almost no data is being captured electronically. Having access to all hospital services from any terminal installed in a public service setting would accomplish this. The department is currently in the process of doing a pilot study of this system; the facility visited will be used as one of the pilot sites. The idea is that if information regarding a particular patient is captured, if $\mathrm{s} /$ he visits another hospital tests do not have to be repeated. It is envisaged that this will save the department lots of money in the long term.

In conclusion, the doctor-nurse team working at this pediatric site has done an enormous amount under difficult circumstances and strategies are in place to ensure that their pediatric ARV services will expand. After the new renovation, the adult ARV clinic will be moved next door to the pharmacy, which is closer to the current pediatric ARV clinic, and everything will be in close proximity to each other. Staff capacity (counselors, dieticians, etc) to assist in the pediatric service will be increased and facilities that include data capturing capabilities will be made available to the pediatric section as well. However, the burden of training and mentorship of staff seems to remain on the shoulders of the current small team.

\section{Non-Governmental District Hospital-McCord Hospital, KwaZulu Natal Province}

\section{Introduction and general overview}

Sinikithemba ("Let's have hope") clinic was founded in 1998. Apparently the founding doctors passed by this beautiful two story house every day, and one day they decided to buy it and start an HIV clinic. The clinic is well supported by the rest of the McCord Hospital governing board. McCord is an NGO hospital that is 60 percent privately funded and 40 percent government funded; therefore, attending patients are expected to pay a fee, though it is less than a normal private hospital fee. The patient clientele is comprised of the more financially advantaged patients. There is an incentive rating assigned to families whereby if they are regular attendees of the support group, one member does not have to pay. For example, by enrolling the entire family, including the father, the family only pays for the couple and the child is free. This method is another way of encouraging entire family HIV testing and is working well at Sinikithemba clinic. The director/coordinator is assisted by a deputy manager for clinic management. To date there are 900 adults and 110 children on ARVs at McCord Hospital. 
McCord Hospital is on a one-way road and each section has a house name. For example, Ridge house is another name for Sinikithemba clinic and Hope house is the psychosocial support department. Inside Sinikithemba clinic there is a large waiting area and barred reception area, with seven consulting rooms, a blood taking area, and a pharmacy. Upstairs is the secretary's quarters, the counseling rooms, and a boardroom. Outside in the courtyard there is a big garden and play area that is supervised on pediatric ARV days. There are also three pre-fabricated counseling rooms that are not soundproof and provide limited privacy. McCord Hospital is on the opposite side from the other houses and from HIVAN, the center for HIV/AIDS networking affiliated to the University of Natal. Within HIVAN is also the director's office, where ongoing computer training is provided for all staff members.

\section{Access to McCord ARV services}

The majority of the patients who access the service are from the areas inside and outside Durban. Patient race, creed, and nationality are varied from what I have observed in the waiting areas inside Ridge and Hope house. Referrals are from other state hospitals in Durban like Prince Mshiyeni, Addington, RK Khan, Osindisweni, Wentwoth, King George, King Edward, and Mahatma Ghandi. The pediatric ward has a 12-bed capacity. The children and mothers identified through the facility's own PMTCT program get preference over all others in terms of prioritization for treatment. A few patients are self-referred.

\section{Pharmacy services}

The ARV service for both adults and pediatrics is run by a full-time pharmacist who is assisted by two part-time pharmacists. The pharmacy is new and construction is still ongoing outside in some consulting areas. There are bars on the outside through which the patients communicate with the pharmacists. Inside it is not very spacious; the smaller room is where all ARVs, TB, and other meds are stored. The fridge is working well, but there is no temperature monitoring log on the fridge. The smaller room is used as a store and has only a lockable door, but no safety gate as yet. Only the full-time pharmacist has a key to the main door and store, and when she is away the key is obtainable from McCord's head pharmacist. Just outside the main door is a small counseling room where the pharmacist does ARV counseling. Patients arrive at the pharmacy with two scripts, a yellow card, and a receipt of payment. One of the two scripts is placed into the patient's folder and the information on the other is captured into a computer and the copy is filed in the pharmacy. The receipt is verified for payment. When there is no receipt, the patient gets sent back to the administrator and an account system may be arranged. The yellow card is a means of tracking which patients belong to Sinikithemba.

There are always two pharmacists checking a script to minimize any erroneous medication dispensing. There is no structured system in place for adherence monitoring. Patients are not asked to bring back empty containers and pills are not counted. Patients do undergo an intensive three-week training; doctors establish who the defaulters are or the potential problem patients in terms of non-adherence, and they are promptly referred to the psychosocial department for ongoing counseling.

The hospital chief pharmacist is the overall procurement officer for all medications, although the fulltime ARV pharmacist completes the pediatric ordering forms on a bi-monthly basis. The ARVs are ordered directly from the pharmaceutical company. The only time they experienced a problem with drug availability was when Zerit was out of stock internationally, which is no longer a problem. 


\section{Psychosocial support groups and linkages}

Support groups are mainly run by the psychosocial department, where the social workers and counselors are the key players spearheading the programs. Once a patient is identified by a nursing sister and the decision is finalized by a doctor, the patient/caregiver is referred to the counselor for three weeks (on consecutive Mondays) of intensive training prior to ARV initiation.

There is a trained social worker in charge of the psychosocial department and he coordinates all counselors and their activities. These activities range from intense adherence counseling and adolescent counseling to support group and sustainable development meetings. As part of the support and sustainable developments, there is a choir made up entirely of HIV-positive choristers. There are small-scale income generation projects, mainly beadwork. Beautiful items, ranging from fashionable jewelry to African garments, are made. The choir and members of the support group travel abroad to market their achievements. The Bambanani camp for children and their parents or caregivers provides additional ongoing support. A support group is held every Tuesday morning from 10am to $1 \mathrm{pm}$, run by a social worker and a psychologist.

Hope House is an apartment with a waiting and reception area where patients are seen by appointment by a member of the psychosocial team. The staff is very friendly and supportive and rooms are brick and soundproof for confidential sessions, unlike the prefabricated counseling rooms outside Ridge House.

\section{Nutritional support}

There is no nutritional support at all at Sinikithemba clinic. There appears to be no need for this service since families attending are in a relatively better socioeconomic position and are not nutritionally challenged, according to staff.

\section{Staffing}

There is a qualified pediatrician working at Sinikithemba clinic who consults on Tuesdays, Thursdays, and Fridays, the pediatric clinic days. There are two other medical officers who consult with both adults and children on pediatric ARV days.

The sister in charge has only been working at Sinekitemba for approximately six months. Although not trained in IMCI, she does not feel apprehensive about furthering her skills in this direction. Her duties mostly encompass overseeing nurse duties and the administration thereof; there are future plans to assist with the pediatric ARV clinic in terms of consulting on pediatric ARV days. The clinic director envisaged that the latter might take another year before it will be in full operation.

The other nurse consults with adult patients about ARVs. She only assists with assessment of children for ARVs and the doctor does another final assessment before the child and caregiver get sent to the counselor to initiate intensive ARV training. Ongoing training to further their HIV/ARV management skills is not a problem at Sinikithemba, and they both feel that they are given lots of opportunities to attend workshops and conferences. There are two staff nurses who take all bloods in a good workable 
space, equipped with all the necessary requirements. Bloods are transported to Global viral laboratory at least twice daily and they do not have any problems with the system.

I have also noticed plenty of student nurses; it is unfortunate that HIV/ARV management does not form part of their training curriculum. None of them are ever assigned to go for mentorship at Sinikithemba for HIV/ARV training.

In conclusion, the staff working at Sinikithemba are the only ones in all provinces I have visited with such few challenges. I think that however small the organization, they have excellent infrastructure and human resource capacity available to deliver a good ARV service to both adults and children. 


\section{Hgrizons}

Horizons is a global operations research program designed to:

- Identify and test potential strategies to improve HIV/AIDS prevention, care, and support programs and service delivery.

- Disseminate best practices and utilize findings with a view toward scaling up successful interventions.

\section{(2) Population Council}

Horizons is implemented by the Population Council in collaboration with

- International Center for Research on Women (ICRW)

- International HIV/AIDS Alliance

- PATH

- Tulane University

- Family Health International (FHI)

- Johns Hopkins University

For more information, please contact:

Horizons Program, Communications Unit 4301 Connecticut Ave, NW Suite 280 Washington, DC 20008 USA

Tel: 202-237-9400

Fax: 202-237-8410

Email: horizons @pcdc.org www.popcouncil.org/horizons 